\title{
NGC 5775: Anatomy of a disk-halo interface
}

\author{
S.-W. Lee ${ }^{1}$, J. A. Irwin ${ }^{2}$, R.-J. Dettmar ${ }^{3}$, C. T. Cunningham ${ }^{4}$, G. Golla ${ }^{3, \star}$, and Q. D. Wang ${ }^{5}$ \\ 1 Astronomy Department, University of Toronto, 60 St. George Street, Toronto, Ontario, Canada M5S 3H8 \\ 2 Department of Physics, Queen's University, Kingston, Ontario, Canada K7L 3N6 \\ e-mail: irwin@astro.queensu.ca \\ 3 Astronomisches Institut der Ruhr-Universität Bochum, Universitätsstr. 150/NA 7, 44780 Bochum, Germany \\ e-mail: dettmar@astro.ruhr-uni-bochum.de \\ 4 National Research Council of Canada, Herzberg Institute of Astrophysics, 5071 West Saanich Road, Victoria, \\ BC, Canada V9E 2E7 \\ e-mail: charles.cunningham@nrc.ca \\ 5 Department of Astronomy, University of Massachusetts, B-524 Lederle Graduate Research Tower, Amherst, \\ MA, 01003, USA \\ e-mail: wqd@astro.umass.edu
}

Received 10 October 2000 / Accepted 12 July 2001

\begin{abstract}
We present the first high-resolution study of the disk-halo interface in an edge-on galaxy (NGC 5775) in which every component of the interstellar medium is represented and resolved (though not all to the same resolution). New single-dish CO $J=2-1$ and CO $J=1-0$ data, ROSAT X-ray data, and HIRES IRAS data are presented along with HI data which emphasizes the high latitude features. In conjunction with previously published radio continuum $(6$ and $20 \mathrm{~cm}$ ) and $\mathrm{H} \alpha$ data, we find spatial correlations between various ISM components in that all components of the ISM are present in the disk-halo features (except for CO for which there is insufficient spatial coverage). The HI features extend to $\sim 7 \mathrm{kpc}$ above the plane, form loops in position-position space, in one case, form a loop in position-velocity space, and are also observed over a large velocity range. This implies that the disk-halo features represent expanding supershells. However, the shells may be incomplete and partially opentopped, suggesting that we are observing the breakup of the supershells as they traverse the disk-halo interface. There is some evidence for acceleration with $z$ and both redshifted and blueshifted velocities are present, although the gas which is lagging with respect to galactic rotation dominates. The radio continuum spectral index is flatter around the shell rims and we show that this cannot be due to a contribution from thermal gas but rather is due to intrinsic flattening of the non-thermal spectral index, suggesting that shocks may be important in these regions. The $\mathrm{H} \alpha$ emission is located interior to the HI. For feature F3, the $\mathrm{H} \alpha$ emission forms the interior "skin" of the HI shell, yet there appears to be a minimum of in-disk star formation immediately below the feature. We present a picture of a "typical" HI supershell which accelerates and breaks up through the disk-halo interface. Such a feature is likely internally generated via an energetic event in the disk.
\end{abstract}

Key words. ISM: bubbles - ISM: general - ISM: structure - galaxies: general - galaxies: individual: NGC 5775 galaxies: spiral

\section{Introduction}

Studies of external edge-on galaxies provide an excellent bird's eye view of galaxy halos and the disk-halo interface without the confusion or distance ambiguities which are

Send offprint requests to: S.-W. Lee, e-mail: swlee@astro.utoronto.ca

* Present address: Gesellschaft für Unix- und Netzwerkadministration, Räterstr. 26, 85551 Kirchheim, Germany

e-mail: golla@genua.de present in our own Milky Way. Such observations require high resolution and good signal-to-noise $(\mathrm{S} / \mathrm{N})$.

When a variety of galaxies is considered, disk-halo activity has been observed in all components of the interstellar medium (ISM), including molecular gas traced by CO (e.g., Irwin \& Sofue 1996), dust as observed in the infrared (e.g. Koo et al. 1991) and optical (e.g., Howk \& Savage 1999), neutral hydrogen (e.g., Irwin \& Seaquist 1990), H $\alpha$ emitting $10^{4} \mathrm{~K}$ ionized gas (e.g., Pildis et al. 1994), the 
radio continuum emitting cosmic ray component (e.g., Hummel et al. 1991) and the X-ray emitting hot coronal gas (e.g., Bregman \& Pildis 1994; Wang et al. 1995; Dahlem et al. 1996) - as well as many other examples. However, little has been done in the way of systematic multiwavelength studies to see how the components relate to each other, to discrete star forming regions in the disk, and to the global properties of the galaxy. To this end, we have undertaken a multi-wavelength study of the edge-on, IR-bright galaxy, NGC 5775, and present here the first comparative study of the disk-halo interface in another galaxy in which every component of the interstellar medium is represented.

NGC $5775\left(D=24.8 \mathrm{Mpc}, H_{0}=75 \mathrm{~km} \mathrm{~s}^{-1} \mathrm{Mpc}^{-1}\right)$ is an edge-on $\left(i=86^{\circ}\right)$ galaxy with a high star formation rate $\left(L_{\mathrm{FIR}}=2.6 \times 10^{10} L_{\odot}\right)$. An optical image of the galaxy is shown in Fig. 1a. NGC 5775 is interacting with its more face-on companion, NGC 5774, 4'.3 to the north-west (NW) from which it appears to be receiving a supply of HI (Irwin 1994), and possibly also with a smaller galaxy, IC 1070, 3'9 to the south-west (SW). Duric et al. (1998) have modeled the global radio continuum halo of NGC 5775 in terms of two components: a broad scale steep spectrum halo with 1 /e scale height of $3.5 \mathrm{kpc}$, and a smaller flatter spectrum component of $\sim 1 \mathrm{kpc}$. Dettmar (1992) made a preliminary comparison of the $\mathrm{H} \alpha$ and radio continuum emission in NGC 5775 and pointed out the spatial coincidence of the $\mathrm{H} \alpha$ filamentary features and the radio continuum spurs. Collins et al. (2000) found the diffuse ionized gas (DIG) layer in NGC 5775 to have a scale height of $800-900$ pc in regions away from any obvious discrete high- $z$ features. They made a comparison of their $\mathrm{H} \alpha$ images with both the radio continuum and total intensity HI, finding a good general correlation in the high- $z$ features in all three components. More recently, an optical emission line study by Rand (2000) shows a rise in the $[\mathrm{OIII}] / \mathrm{H} \alpha$ line ratio with $z$, indicating that photoionization at a single temperature cannot alone be producing the emission; shocks and/or a change in temperature is required. A decrease in rotational velocity with $z$ is also observed, with the highest latitude gas showing essentially no rotation.

In this paper, we present new CO $J=1-0$ and CO $J=2-1$ observations, new HIRES infra-red images, and a new X-ray image of NGC 5775. We also present new results on the $\mathrm{HI}$ distribution using the same data as in Irwin (1994) but dealing more specifically with the high latitude gas. In addition, we present an overview of previous results from other wavebands, highlighting features which may be related to the interaction, the in-disk distribution, and the high latitude and halo emission. Since the data are voluminous, in this paper, we focus primarily on spatial correlations of the different ISM components. We also pay special attention to the high- $z$ features and their relationship with the distributions in the disk. As the data are not all to the same resolution, we compare components which are most similar in resolution to each other.

\section{Observations and data reduction}

\subsection{Radio continuum}

The radio continuum observations are taken from Duric et al. (1998) and were obtained with the Very Large Array (VLA) over several years at both 6 and $20 \mathrm{~cm}$ wavelengths in several configurations of the array. Selected images, which have been cleaned and selfcalibrated, are shown in Figs. 1 and 2, i.e., a $20 \mathrm{~cm}$ $\mathrm{B}+\mathrm{C}+\mathrm{D}$ Array $20 \mathrm{~cm}$ image at $23^{\prime \prime}$ resolution (Fig. 1b), a $6 \mathrm{~cm} \mathrm{C}+\mathrm{D}$ array image at $15^{\prime \prime}$ resolution (Fig. 2a), a $20 \mathrm{~cm} \mathrm{~B}+\mathrm{C}+\mathrm{D}$ array image at $15^{\prime \prime}$ resolution (Fig. 2b) and a $20 \mathrm{~cm} \mathrm{~B}$ array image at $5^{\prime \prime}$ resolution (Fig. 2d). The spectral index map made from the 6 and $20 \mathrm{~cm}$ maps at $15^{\prime \prime}$ resolution is also presented in Fig. 2c. The rms noise levels are indicated in the figure caption.

\subsection{Neutral hydrogen}

Neutral hydrogen data are taken from Irwin (1994) and were obtained using the VLA in its $\mathrm{B} / \mathrm{C}$ configuration with a velocity resolution of $41.7 \mathrm{~km} \mathrm{~s}^{-1}$. Two (Right Ascension - Declination - Velocity) cubes were made, representing naturally and uniformly weighted data, with resulting rms noise per channel of 0.4 and $0.5 \mathrm{mJy}_{\text {beam }}{ }^{-1}$, respectively. The integrated intensity (zeroth moment) maps made from these data are presented in Fig. 1c (natural weight) and Fig. 1d (uniform weight). The most obvious $\mathrm{HI}$ extensions are labelled F1 to F3 in Fig. 1d.

\subsection{Far infra-red (FIR)}

Four HI-RES infra-red images (at 12, 25, 60 and $100 \mu \mathrm{m}$ ) were obtained by request to the Infra-red Processing and Analysis Center (IPAC). The HI-RES images were obtained from the Infra-Red Astronomical Satellite (IRAS) observations using the Maximum Correlation Method as described in Aumann et al. (1990). This algorithm makes use of the fact that the spatial separation between flux measurements ("footprints") is typically less than the full width half maximum ( $F W H M$ ) of any individual detector, i.e. the sources have been oversampled. The reconstructed beam FWHMs constitute improvements by factors of 3.8 , $3.7,3.5$, and 2.6 over the mean FWHMs of the individual detectors for the 12, 25, 60, and $100 \mu \mathrm{m}$ bands, respectively. The HI-RES maps, the result of 20 iterations of the algorithm, are shown in Figs. 3a to d.

\section{4. $H_{\alpha}$}

The $\mathrm{H} \alpha$ image was obtained from the European Southern Observatory (ESO) $3.5 \mathrm{~m}$ New Technology Telescope (NTT). The integration time was $60 \mathrm{~min}$ in a filter of $F W H M=75 \AA$ and the seeing was $1^{\prime \prime} 1$. The H $\alpha$ image was obtained after $R$-band subtraction and is shown in Fig. 4a. Calibration was done using an $R$-band magnitude of NGC 5774 and the level of the rms noise of the map 


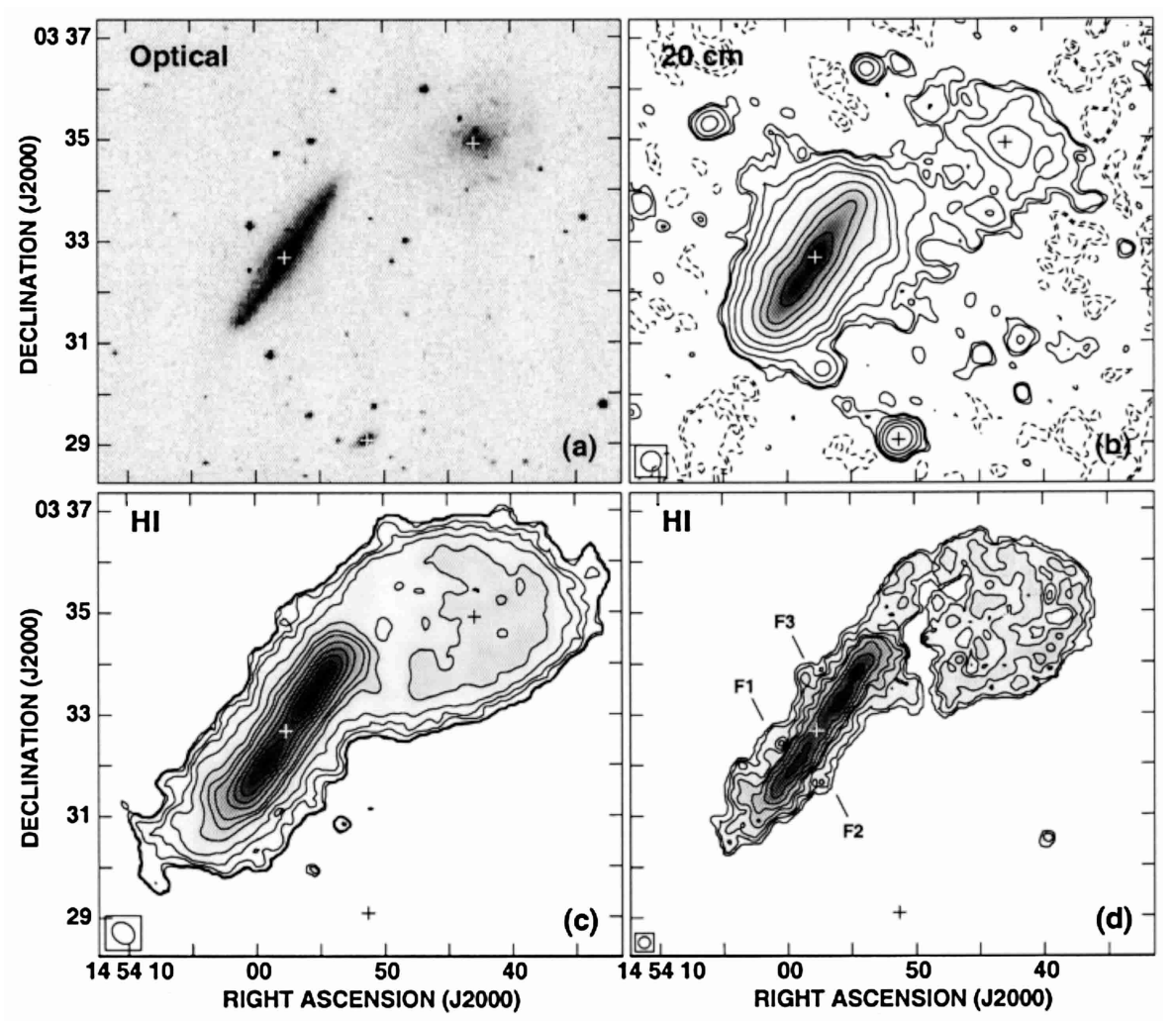

Fig. 1. Sequence of images of NGC 5775 (edge-on), NGC 5774 (nearly face-on, to the north-west), and IC 1070 (to the south). The optical positions of each galaxy, from the NASA/IPAC Extragalactic Data Base (NED), are marked with white or black crosses. The synthesized beam (if applicable) is shown at the bottom left of each image. a) Optical image from the Space Telescope Science Institute (STScI) Digitized Sky Survey (DSS). b) VLA $20 \mathrm{~cm}$ radio continuum image from combined B, C, and D array data (see Duric et al. 1998). The synthesized beam is $23^{\prime \prime} .5 \times 23^{\prime \prime} 4$ at a position angle (PA) of $45^{\circ} .2$. The rms noise is $43 \mu \mathrm{Jy}_{\mathrm{beam}}{ }^{-1}$, the peak is $34.9 \mathrm{mJy} \mathrm{beam}^{-1}$ and contour levels are at $\pm 0.065, \pm 0.1, \pm 0.2,0.4,0.75,2.0,5.0,10$, 20, and $34 \mathrm{mJy}$ beam $^{-1}$. c) Column density map of the naturally weighted neutral hydrogen emission (see Irwin 1994). The beam is $28^{\prime \prime} .8 \times 22^{\prime \prime} .8$ at $\mathrm{PA}=52^{\circ} .0$, the peak is $72 \times 10^{20} \mathrm{~cm}^{-2}$, and contours are at $0.5,1,2,3,5,10,15,20,30,40,50,60$ and $70 \times 10^{20} \mathrm{~cm}^{-2}$. d) As in c) but using uniformly weighted data. The beam is $13^{\prime \prime} .6 \times 13^{\prime \prime} 4$ at PA $=-33.7$, the peak is $100 \times 10^{20} \mathrm{~cm}^{-2}$ and contours are at $1,5,10,17.5,25,40,60$, and $82.5 \times 10^{20} \mathrm{~cm}^{-2}$. HI extensions are labelled F1 to F3.

is $6.6 \times 10^{-18} \mathrm{ergs} \mathrm{s}^{-1} \mathrm{~cm}^{-2} \operatorname{arcsec}^{-2}$. Thus, this image is of comparable sensitivity to that of Collins et al. (2000). There is also a background gradient at about the level of the noise. This image, without the quantitative scale, has previously been presented in Dettmar (1992).

\section{5. $\mathrm{CO}$}

The molecular gas in the central region of the galaxy was mapped in the CO $J=1-0$ line using the $15 \mathrm{~m}$ SwedishESO Submillimetre Telescope (SEST) ${ }^{1}$ during July 27-31 1990 and in the CO $J=2-1$ line using the James Clerk Maxwell Telescope (JCMT) ${ }^{2}$ during the period from

1 The SEST is operated jointly by ESO and the Swedish National Facility for Radio Astronomy, Onsala Space Observatory, Chalmers University of Technology.

2 The JCMT is operated by the Joint Astronomy Centre in Hilo, Hawaii on behalf of the parent organizations Particle Physics and Astronomy Research Council in the United Kingdom, the National Research Council of Canada and The Netherlands Organization for Scientific Research.
July 1993 to February 1995. The half-power-beam-width (HPBW) of the SEST at $115 \mathrm{GHz}$ is $43^{\prime \prime}$ and that of the JCMT at $230 \mathrm{GHz}$ is $21^{\prime \prime}$. Pointing accuracies are roughly $5^{\prime \prime}$ at both telescopes. At the JCMT, the absolute flux calibration was checked against sources of known flux during each observing run and was found to be good to within $20 \%$. Spectra at 19 points for CO $J=1-0$ and 36 points for CO $J=2-1$ were integrated over velocity and interpolated onto a map grid. The resulting maps are shown in Figs. 4b and c. Full details are presented in Lee (1998). The results of a line ratio analysis will be presented in a separate paper.

\subsection{X-ray}

X-ray observations were obtained from the ROSAT satellite (sequencenumber $=$ RP800478N00) between July 29 and August 13, 1993. The total exposure time was $6140 \mathrm{~s}$ and the angular resolution is about $30^{\prime \prime}$. An overlay of these results on an optical image is shown in Fig. 5. The X-ray spectrum is characterized by an optically-thin 

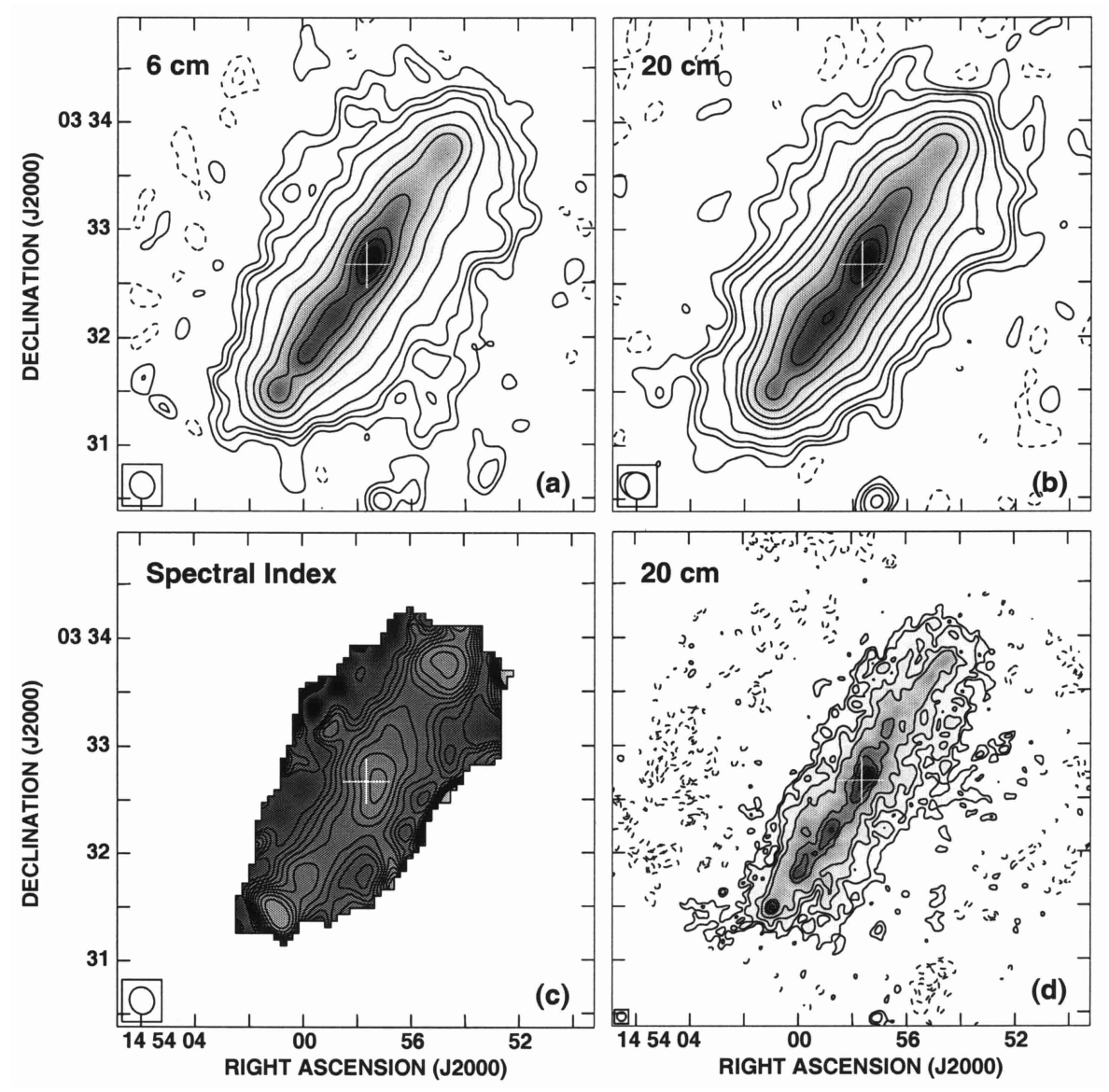

Fig. 2. a) VLA $6 \mathrm{~cm}$ radio continuum image of NGC 5775 from combined C and D array data (see Duric et al. 1998). The restoring beam is $15^{\prime \prime} 26 \times 13^{\prime \prime} .50$ at $\mathrm{PA}=20^{\circ} 07$. The map peak is $8.7 \mathrm{mJy}_{\text {beam }}{ }^{-1}$ and rms noise is $20 \mu \mathrm{Jy}_{\text {beam }}{ }^{-1}$. Contour levels are at $\pm 0.03, \pm 0.06,0.09,0.15,0.3,0.75,1.5,3.0,4.5,6.0$, and $7.5 \mathrm{mJy}_{\text {beam }}{ }^{-1}$. b) VLA 20 cm radio continuum image of NGC 5775 from combined B, C and D array data (see Duric et al. 1998). The restoring beam is $15^{\prime \prime} .26 \times 13^{\prime \prime} 50$ at PA $=20^{\circ} 07$. The map peak is $19.1 \mathrm{mJy}_{\text {beam }}{ }^{-1}$ and $\mathrm{rms}$ noise is $25 \mu \mathrm{Jy}_{\text {beam }}{ }^{-1}$. Contour levels are at $\pm 0.07,0.14,0.21,0.35,0.7,1.05,1.75$, 3.5, 7.0, 10.5, and $14 \mathrm{mJy}_{\text {beam }}{ }^{-1}$. c) The spectral index map made by combining the maps from $2 \mathrm{a}$ and $2 \mathrm{~b}$ (see Duric et al. 1998). The spectral index, $\alpha$, is defined according to the $\mathrm{S}(\nu) \propto \nu^{-\alpha}$ convention. The contours start at 0.6 (light grey) to 1.0 (black) with an increment of 0.05. d) VLA $20 \mathrm{~cm}$ radio continuum image of NGC 5775 from the B array data (see Duric et al. 1998). The restoring beam is $5^{\prime \prime} .03 \times 4^{\prime \prime} .96$ at $\mathrm{PA}=-41^{\circ} .48$. The map peak is $4.2 \mathrm{mJy} \mathrm{beam}^{-1}$ and $\mathrm{rms}$ noise is $30 \mu \mathrm{Jy}$ beam ${ }^{-1}$. Contour levels are at $\pm 0.09,0.15,0.3,0.6,1.5,2.1$, and $3.0 \mathrm{mJy}_{\text {beam }}{ }^{-1}$.

thermal plasma with average temperature of $0.4 \mathrm{keV}$ (about 5 million $\mathrm{K}$ ). The limited spatial resolution and counting statistics of the X-ray data prevent us from a spatially-resolved spectral analysis. Thus a significant contribution of point-like sources, which typically have relatively hard X-ray spectra, in the galactic disk region may be important.

\section{Results}

\subsection{The interaction}

\subsubsection{Tidal bridges}

The environment of NGC 5775 is illustrated in Fig. 1. The interaction of NGC 5775 with its companion, NGC 5774, is indicated by the presence of two bridges. The bridges are especially apparent in HI (Figs. 1c and d) and the HI velocity field suggests mass transfer from NGC 5774 to NGC 5775 (Irwin 1994). There is no strong optical disturbance in either galaxy (Fig. 1a), but faint emission is seen along both bridges on the Palomar Observatory Sky Survey (POSS) blue print indicating that a stellar component is indeed present. Although not shown here, our $\mathrm{H} \alpha$ image shows emission along the southern bridge (also confirmed by Collins et al. 2000). If the ionization is due to hot young stars, then star formation must be occurring in the bridge itself since the lifetimes of such stars are shorter than the interaction timescale (see Irwin \& Caron 1994). Another tracer of hot young stars, non-thermal radio continuum emission, also occurs along the southern bridge (Fig. 1b). Note that, when the resolution is sufficient, considerable structure is observed in the bridges.

An overlay of radio continuum (from Fig. 1b, rotated) as well as the HIRES $12 \mu \mathrm{m}$ and $25 \mu \mathrm{m}$ (from 


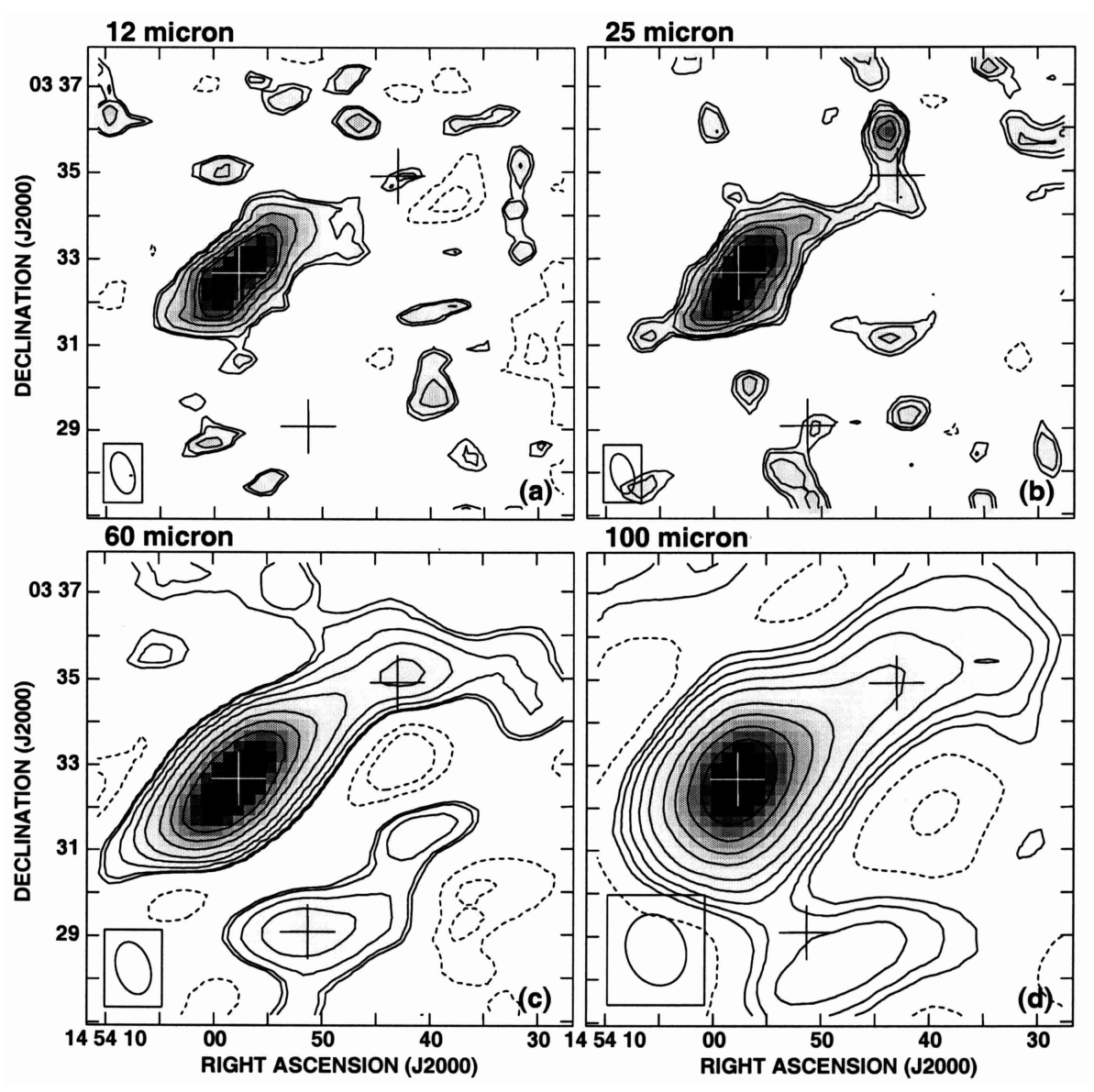

Fig. 3. As in Fig. 1 but showing the HIRES images. a) $12 \mu \mathrm{m}$ image. The beam is $59^{\prime \prime} 4 \times 30^{\prime \prime} 6$ at PA $=15.6$. The map peak is 11.5 $\mathrm{MJy} \mathrm{sr}^{-1}$ and the rms noise is $0.29 \mathrm{MJy} \mathrm{sr}^{-1}$. Contours are at $\pm 0.44, \pm 0.58,1.0,1.8,3.0,4.0$, and $7.0 \mathrm{MJy} \mathrm{sr}^{-1}$. b) $25 \mu \mathrm{m}$ image. The beam is $57^{\prime \prime} 6 \times 30^{\prime \prime} 6$ at $\mathrm{PA}=15$. 7 . The peak is $14.1 \mathrm{MJy} \mathrm{sr}^{-1}$ and $\mathrm{rms}$ noise is $0.38 \mathrm{MJy} \mathrm{sr}^{-1}$. Contours are at \pm 0.57 , $0.8,1.2,2.3,3.5,5.7$ and $9.0 \mathrm{MJy} \mathrm{sr}^{-1}$. c) $60 \mu \mathrm{m}$ image. The beam is $75^{\prime \prime} 0 \times 466^{\prime \prime} 8$ at PA $=16.8$. The peak is $128.8 \mathrm{MJysr}^{-1}$ and $\mathrm{rms}$ noise is $0.14 \mathrm{MJy} \mathrm{sr}^{-1}$. Contours are at $\pm 0.24, \pm 0.33,0.65,1.3,2.6,6.4,13,26$ and $58 \mathrm{MJy} \mathrm{sr}^{-1}$. d) $100 \mu \mathrm{m}$ image. The beam is $101^{\prime \prime} 4 \times 82^{\prime \prime} .2$ at $\mathrm{PA}=19.8$. The peak is $200.4 \mathrm{MJy} \mathrm{sr}^{-1}$ and $\mathrm{rms}^{\circ}$ noise is $0.15 \mathrm{MJy} \mathrm{sr}^{-1}$. Contours are at \pm 0.23 , $\pm 0.50,1.0,2.0,4.0,10,20,40,80$ and $125 \mathrm{MJy} \mathrm{sr}^{-1}$.

Figs. 3a and b) on the HI image (from Fig. 1c) is shown in Fig. 6. Particularly interesting is the FIR emission between the galaxies which, at $12 \mu \mathrm{m}$ and $25 \mu \mathrm{m}$, occurs only along the southern bridge. At $12 \mu \mathrm{m}$ (Fig. 6b), the emission is seen as a two-pronged spur extending from NGC 5775 with the southern prong the strongest. At $25 \mu \mathrm{m}$ (Fig. 6c), a complete bridge connects the galaxies. This bridge starts as a strong spur from NGC 5775 (at $\mathrm{RA}=14^{\mathrm{h}} 53^{\mathrm{m}} 51^{\mathrm{s}}, \delta=03^{\circ} 33^{\prime} 49^{\prime \prime}$ ) which corresponds to a radio continuum spur (Fig. 6a, third contour). However, the FIR bridge is offset $\approx 30^{\prime \prime}$ to the NE (i.e. towards the inside) of the centroid of the $\mathrm{HI} /$ radio continuum bridge. Together with the two-pronged spur at $12 \mu \mathrm{m}$, this suggests that the dust could be in a sheath around the radio continuum emission. Temperature gradients and/or different dust properties may also be contributing to the different morphologies seen between the IR wavebands. In lower resolution $60 \mu \mathrm{m}$ and $100 \mu \mathrm{m}$ images (Fig. 3), the two galaxies appear enveloped in emission.
The X-ray emission (Fig. 5) shows two bright peaks north and south of the companion galaxy, NGC 5774, and another weaker peak to the east of it. The bright emission peaks are most likely background active galactic nuclei or foreground stars. The fainter peak occurs within the northern bridge, at RA $=14^{\mathrm{h}} 53^{\mathrm{m}} 50 \mathrm{~s} .7, \delta=03^{\circ} 35^{\prime} 10^{\prime \prime}$, and is near, but not exactly coincident with a decrease in the HI emission. If this peak is caused by HI absorption of the X-ray emission in the bridge, this would place NGC 5774 on the near side of the hot gas. This is consistent with the fact that NGC 5774 is blueshifted with respect to NGC 5775 and may be in front of it.

It would appear that the interaction is causing various components in NGC 5774, including both stars and the ISM, to be transferred to NGC 5775 through two bridges. There is evidence for star formation in the southern, but not the northern, bridge. Thus the interaction has either been sufficiently disruptive that it has induced star formation in the bridge, or it has been sufficiently quiescent that 


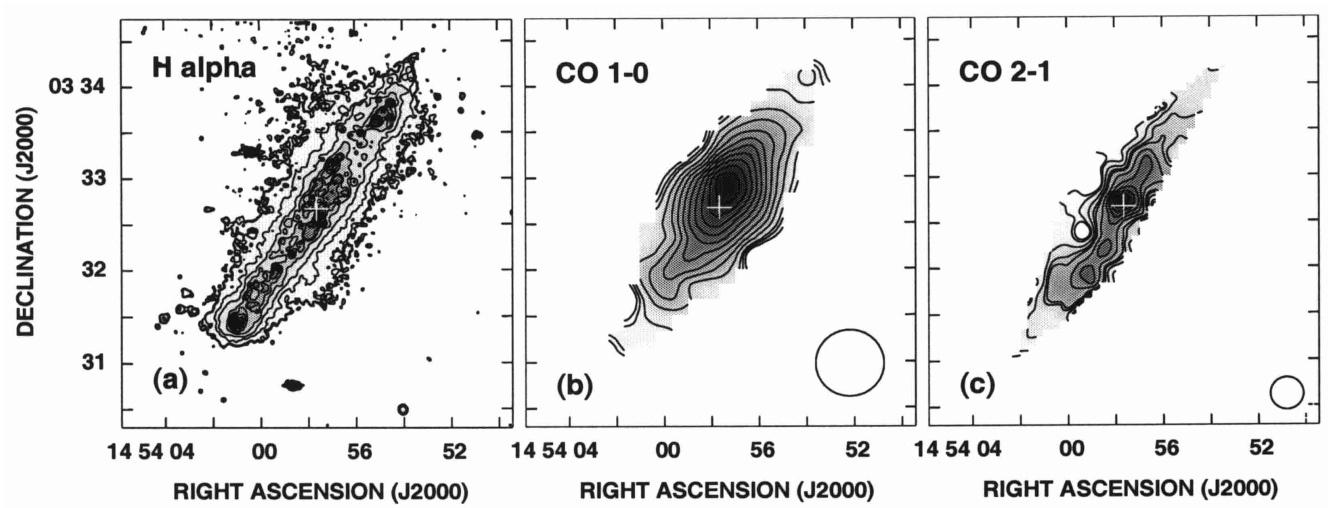

Fig. 4. a) The continuum-subtracted $\mathrm{H} \alpha$ image of NGC 5775. Seeing is about $1^{\prime \prime}$. Contours are 4, 5, 10, 20, 50, 100, 150, 250, $500 \times 6.6 \times 10^{-18} \mathrm{ergs} \mathrm{s}^{-1} \mathrm{~cm}^{-2} \operatorname{arcsec}^{-2}$. The white cross marks the optical position of the galaxy. b) ${ }^{12} \mathrm{CO} J=1-0$ integrated intensity map of NGC 5775 obtained at the SEST. The beam size is $43^{\prime \prime}$ and the map peak is $22.6 \mathrm{~K} \mathrm{kms}^{-1}$. Contour levels are at $1,2,3,5,7,9,11,13,15,17,19$ and $21 \mathrm{~K} \mathrm{kms}^{-1}$. c) ${ }^{12} \mathrm{CO} J=2-1$ integrated intensity map of NGC 5775 obtained at the JCMT with a beam size of $21^{\prime \prime}$. The map peak is $40.0 \mathrm{~K} \mathrm{kms}^{-1}$. Contour levels are at 1, 2, 5, 8, 10, 15, 20, 25, 30 and $35 \mathrm{~K} \mathrm{kms}^{-1}$.

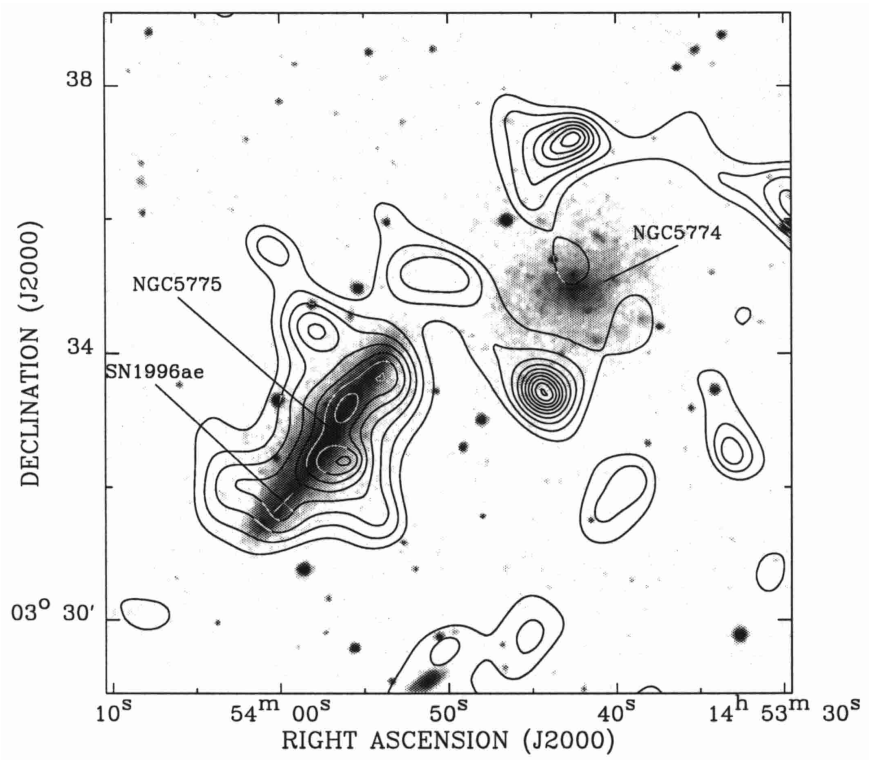

Fig. 5. ROSAT X-ray image of NGC 5775 and its environment superimposed on an optical image.

star forming regions previously present in NGC 5774 may simply have continued their activity through the bridge as they transfer to NGC 5775. Given the continuity of the ISM in NGC 5774 into the bridges and the lack of any strong optical disturbance in the galaxies, the latter may be the case. Follow-up observations of the ionized regions in the bridge would be useful to confirm whether they indeed trace star forming regions, or whether shocks and collisionally ionized gas are present.

\subsubsection{South-east feature (Tidal tail?)}

At the far south-east tip of NGC 5775, there is a feature which may be tidal in origin. It is most obvious in the $\mathrm{H} \alpha$ map (Fig. 4a) at $\alpha=14^{\mathrm{h}} 54^{\mathrm{m}} 03^{\mathrm{s}}, \delta=03^{\circ} 31^{\prime} 30^{\prime \prime}$, where an arc-like string of HII regions extends abruptly away from the end of the disk. The $\mathrm{H} \alpha$ emission is located along the northern ridge of a radio continuum extension in roughly the same direction (Figs. 2b and d). The feature can also be seen as a small extension in neutral hydrogen (Figs. 1c and d) and as a south-east "knob" of FIR emission on the $25 \mu \mathrm{m}$ map (Fig. 6c). As with the bridge, the FIR emission is displaced slightly from the HI emission.

\subsubsection{IC 1070}

The smaller companion galaxy, IC 1070, has been detected in the radio continuum (Fig. 1b), infrared (Figs. 3c and d), and probably in X-ray as well (Fig. 5) but not in neutral hydrogen. These images suggest that this galaxy may also be taking part in the interaction.

\subsection{Disk emission and star forming regions}

In Fig. 7, contours of the disk emission from the different ISM components are superimposed on the $\mathrm{H} \alpha$ greyscale image (except for Fig. 7a where $\mathrm{H} \alpha$ contours are superimposed on the $R$ band image). The CO, radio continuum, and FIR emission (Fig. 3) all peak at or near the nucleus of NGC 5775, while the HI occurs in a ring with a central depression. The optical and $\mathrm{H} \alpha$ images show a prominent dust lane along the eastern side of the major axis (top in Fig. 7), indicating that this is the closer side. The north-west (NW) major axis is advancing and the south-east (SE) side is receding, so trailing spiral arms will have an integral sign $\left(\int\right)$ shape for which there is some evidence in the $\mathrm{H} \alpha$ (Fig. 7a), high resolution radio 


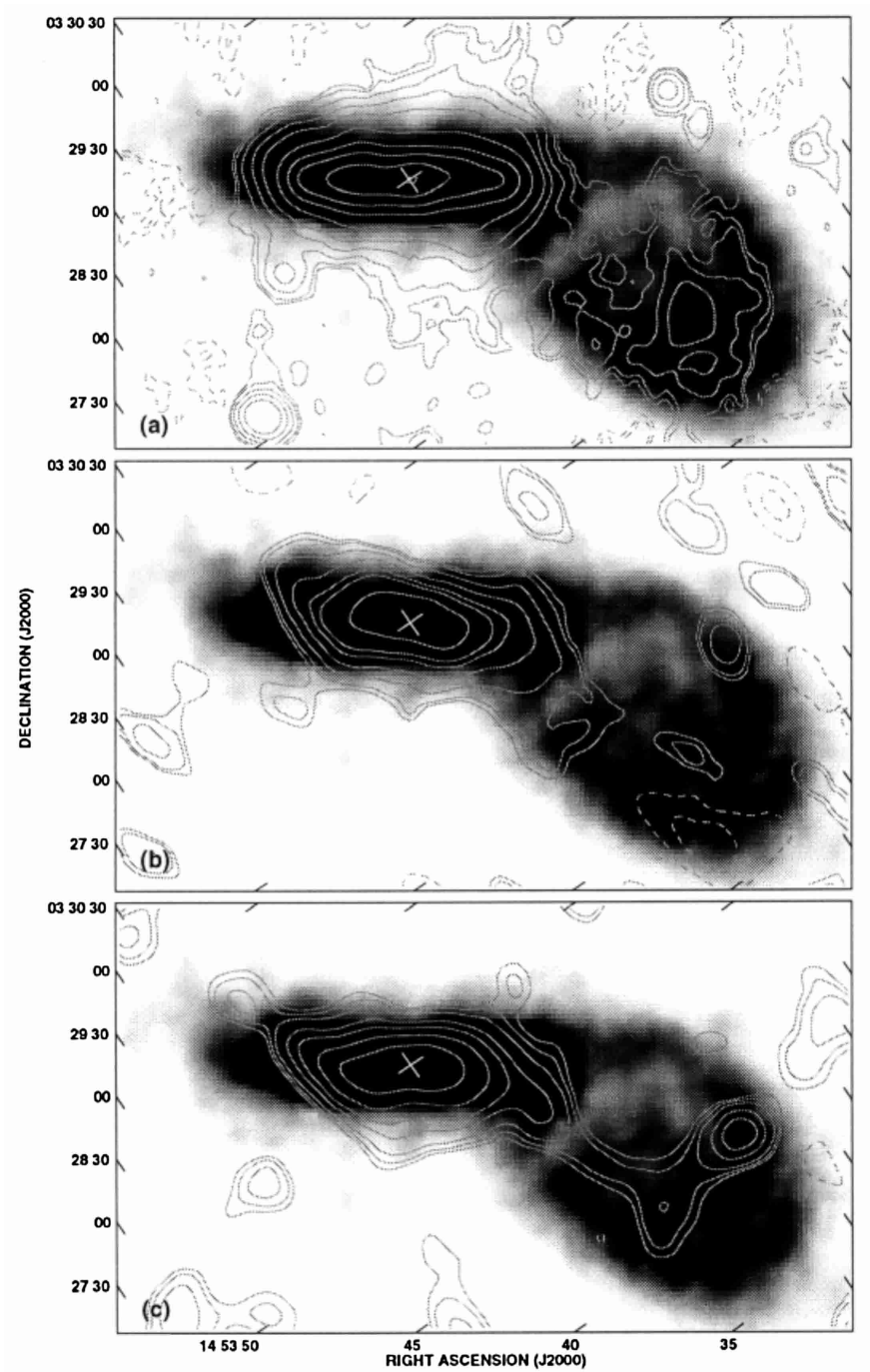

Fig. 6. a) $23^{\prime \prime}$-resolution $20 \mathrm{~cm}$ radio continuum contours superimposed on the natural weighting HI total intensity image. Contours are as in Fig. 1b. b) IRAS $12 \mu \mathrm{m}$ contours (as in Fig. 3a) superimposed on the natural weighting HI total intensity image. c) IRAS $25 \mu \mathrm{m}$ contours (as in Fig. 3b) superimposed on the natural weighting HI total intensity image.

continuum (Fig. 7b), and CO $J=2-1$ (Fig. 7e) distributions.

The 3 brightest knots of $\mathrm{H} \alpha$ emission, which we take to represent the brightest star forming regions, are labelled A, B, and C (brighter to dimmer) in Fig. 7a. The first, Feature A, is very compact and shows the best correspondence with a radio continuum peak (Fig. 7b). It is located at the far tip of the SE major axis $\left(\alpha=14^{\mathrm{h}} 54^{\mathrm{m}} 1.1\right.$, $\left.\delta=03^{\circ} 31^{\prime} 26^{\prime \prime}\right)$. Feature B is the brightest star forming region near the nucleus, located $6^{\prime \prime}$ south of the nucleus (at RA $\left.=14^{\mathrm{h}} 53^{\mathrm{m}} 57^{\mathrm{s}} 6, \delta=03^{\circ} 32^{\prime} 34^{\prime \prime}\right)$ and slightly offset from the radio continuum peak (at $\alpha=14^{\mathrm{h}} 53^{\mathrm{m}} 57^{\mathrm{s}} 4$, $\delta=03^{\circ} 32^{\prime} 43^{\prime \prime}$; average of $20 \mathrm{~cm}$ and $6 \mathrm{~cm} 5^{\prime \prime}$-resolution images), likely due to dust obscuration. Feature $\mathrm{C}$ is located at the far tip of the NW major axis (at $\alpha=$ $14^{\mathrm{h}} 53^{\mathrm{m}} 54.5, \delta=03^{\circ} 33^{\prime} 49^{\prime \prime}$ ) at the end of a string of several HII regions where the radio continuum emission is also stronger. Several other HII regions are also visible throughout the disk.

Generally, there is quite good agreement between the $5^{\prime \prime}$ resolution $20 \mathrm{~cm}$ radio continuum emission and the $\mathrm{H} \alpha$ emission (Fig. 7b), with peaks occurring at roughly the same place in both maps (a few offsets are also seen). The CO $J=2-1$ distribution also correlates with these components (Fig. 7e), consistent with the fact that these are each star formation tracers. The spectral index map (Fig. 7c) also shows a strong correlation with regions of star formation in the sense that the spectral index is significantly lower $(\sim 0.6)$ in these regions. Only the disk HI distribution (Fig. $7 \mathrm{~d}$ ) shows little correlation with the other bands.

Both $\mathrm{H} \alpha$ and radio continuum distributions have lower intensities about $50^{\prime \prime} \mathrm{NW}$ of the optical nucleus between B and $\mathrm{C}$, near $\alpha=14^{\mathrm{h}} 53^{\mathrm{m}} 55^{\mathrm{s}} .5, \delta=03^{\circ} 33^{\prime} 20^{\prime \prime}$. The CO distribution also falls off strongly as this depression is approached. The decline in intensity is most obvious in $\mathrm{H} \alpha$ in which there appears to be a "gap" in the emission. The fact that the radio continuum and molecular components correlate suggests that this $\mathrm{H} \alpha$ gap is not due to dust obscuration. Within this region there are also several faint HII regions, one of which is clearly extraplanar (see Sect. 3.3.2). At the corresponding offset on the SE disk between A and B near $\alpha=14^{\mathrm{h}} 53^{\mathrm{m}} 59^{\mathrm{s}}, \delta=03^{\circ} 32^{\prime} 00^{\prime \prime}$, a similar depression in the $\mathrm{H} \alpha$ distribution can be seen. Here, the radio continuum and $\mathrm{CO}$ emission do not fall off as dramatically, suggesting that dust obscuration may be contributing more strongly to the $\mathrm{SE} \mathrm{H} \alpha$ decrement.

\subsection{Extraplanar features}

NGC 5775 shows numerous extensions, arcs, loops and other connecting features between the disk and halo in every component of the interstellar medium. Even CO, which has not been fully mapped to high galactic latitudes at the required sensitivity and resolution, shows some evidence for vertical extensions (Fig. 7e) near the nucleus.

\subsubsection{High latitude neutral hydrogen}

Of the extraplanar emission seen over the various wavebands, it is the high latitude HI features which are the most well-defined and so we use these as a reference for comparison with other wavebands. HI features in this galaxy have been pointed out by Irwin (1994) and presented in detail in Lee (1998).

A total of $6 \mathrm{HI}$ high-latitude features have been identified (Lee 1998) by visually inspecting the velocity channel maps. Only features that appear at the same spatial location in at least two consecutive velocity channels were considered to be real. In this paper, we concentrate on the three most obvious features, labelled F1 to F3 in Fig. 1d. All three can be traced for many channels and reach projected heights of 7.1, 6.2, and $7.3 \mathrm{kpc}$ from the midplane for F1, F2 and F3, respectively. F1 and F2 appear to be 


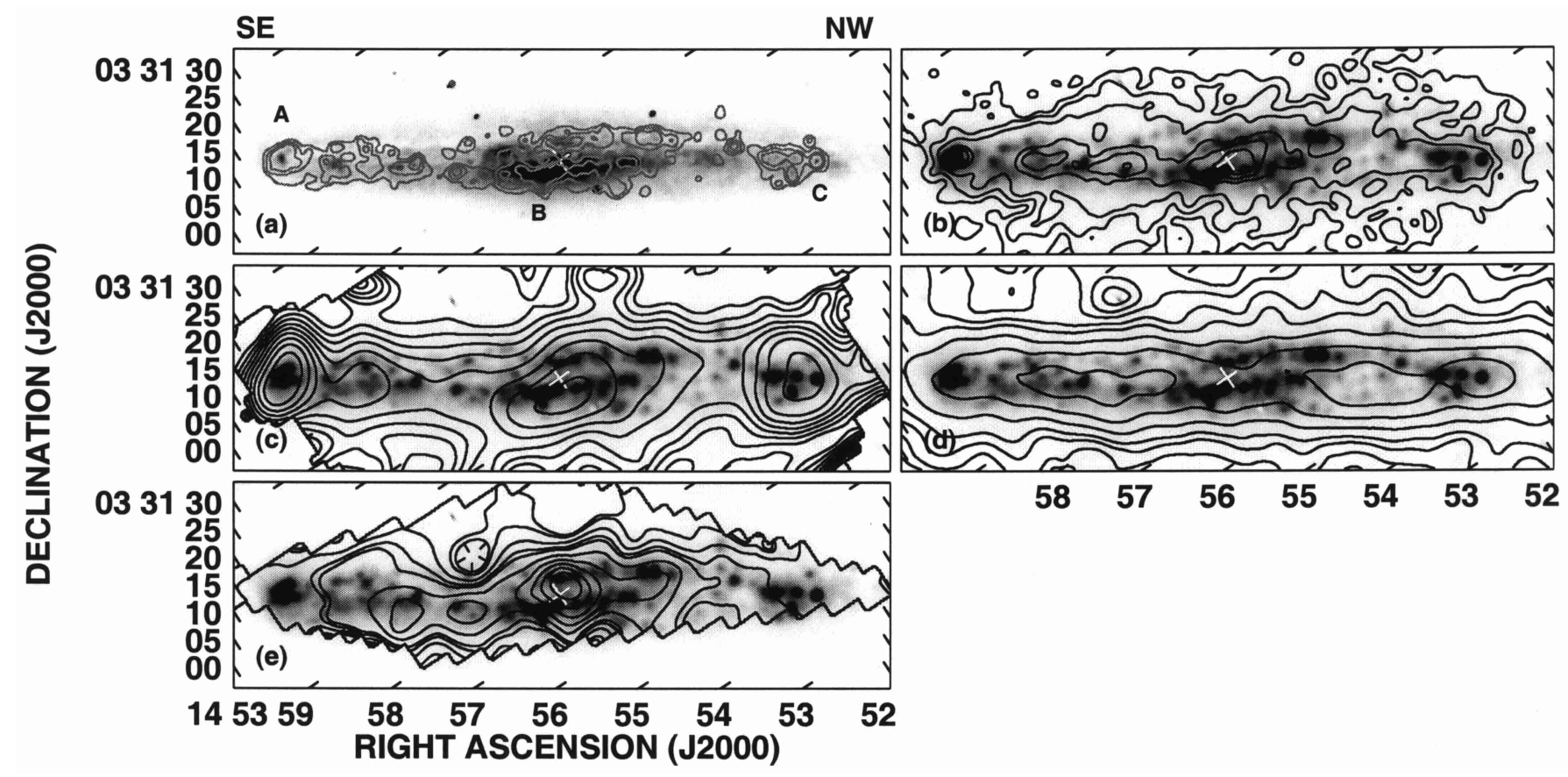

Fig. 7. Comparisons of the in-disk distributions in various wavebands. a) $H \alpha$ contours superimposed on the $R$-band image. Contours are at 70, 120 and 200 times $6.6 \times 10^{-18} \mathrm{ergs} \mathrm{s}^{-1} \mathrm{~cm}^{-2} \operatorname{arcsec}^{-2}$. The features labelled A, B, and C are the brightest $\mathrm{H} \alpha$ emission in the disk . b) $5^{\prime \prime}$-resolution $20 \mathrm{~cm}$ radio continuum contours superimposed on the H $\alpha$ greyscale. c) Radio continuum spectral index (15"-resolution) superimposed on $\mathrm{H} \alpha$. d) Uniformly-weighted HI total intensity (13"'-resolution) over $\mathrm{H} \alpha$. e) ${ }^{12} \mathrm{CO} J=2-1$ integrated intensity (21"

roughly symmetrically placed with respect to the galactic centre. Because of the spatial and velocity coherence of the features, because they appear loop-like (or arc-like with open tops), and because they show expansion (see below), we identify them as HI supershell candidates or remnants, similar to those found in the Milky Way (Heiles 1979; Heiles 1984). Their radii, $R_{\mathrm{sh}}$, projected galactocentric distances, $D_{\text {sh }}$, half the velocity range over which they are observed, $V_{\mathrm{sh}}$, and number densities at midplane, $n_{\mathrm{o}}$, are given in Table 1 . Note that $n_{\mathrm{o}}$ is calculated based on the HI modeled density distribution given in Irwin (1994), assuming that the galactocentric radius is equivalent to the projected galactocentric radius. It is interesting that the most well-defined extra-planar features occur, not near the nucleus, but at projected radii greater than $4.3 \mathrm{kpc}$, indicating that their actual galactocentric radii are greater than or equal to these values.

Feature F2 shows the clear signature, in positionvelocity (PV) space, of an expanding supershell (see Fig. 9h and Sect. 3.5.2). While the other features show no clear PV expansion signature, we can infer that expansion is taking place since the velocity range over which the feature is seen (hundreds of $\mathrm{km} \mathrm{s}^{-1}$ ) is greater than the change in velocity of the underlying rotation curve over the diameter of the feature at its galactocentric radius (less than $25 \mathrm{~km} \mathrm{~s}^{-1}$, Irwin 1994). In addition, double line structure is also seen (Sect. 3.5). Note also that there is no confusion against the disk at these high latitudes. We can therefore estimate a kinematic age of the shells, $\tau_{\mathrm{sh}}=R_{\mathrm{sh}} / V_{\mathrm{sh}}$. The results (Table 1 ) are of order $10^{7} \mathrm{yr}$, similar in magnitude as those found in NGC 3079 (Irwin \& Seaquist 1990) and NGC 4631 (Rand \& van der Hulst 1993).

If the supershells are produced by supernova explosions in the disk, we can also estimate the input energies required to form them. For an expanding shell which is formed from a one-time energy injection and which is now in the radiating phase of its evolution, the required energy injected (Chevalier 1974) is $E_{\mathrm{E}}=5.3 \times 10^{43} n_{0}^{1.12} R_{\mathrm{sh}}^{3.12} V_{\mathrm{sh}}^{1.4}$ where $n_{0}$ is the number density at midplane in $\mathrm{cm}^{-3}$, $R_{\text {sh }}$ and $V_{\text {sh }}$ are the radius and expansion velocity of the shell in pc and $\mathrm{km} \mathrm{s}^{-1}$, respectively. Chevalier's numerical models show that $<10 \%$ of the explosion energy is transferred to kinetic energy of the expanding supershells. The results, making use of the modeled number density profile given in Irwin (1994) are shown in Table 1. For a typical supernova energy of $10^{51} \mathrm{ergs}$, the energy input required for $\mathrm{F} 1$ alone $\left(1.9 \times 10^{55} \mathrm{ergs}\right)$ would require $\approx 10^{4}$ correlated supernovae within $\approx 10^{7} \mathrm{yr}$ which is also roughly the age of a typical young star cluster or OB association.

If we consider a slower, continuous energy injection over a typical cluster $(\sim$ shell $)$ age of several $\times$ $10^{7}$ years, the energy required can be expressed as $E_{\mathrm{c}}=1.16 \times 10^{41} R_{\mathrm{sh}}^{5} n_{0} t_{7}^{-3}$ ergs (from Eq. (3) in McCray \& Kafatos 1987). These results are also listed in Table 1. Therefore, the energy needed to form each supershell, be it a one-time energy injection, or a continuous supply, is extraordinarily large. NGC 5775 has 
Table 1. Parameters for Supershells in NGC 5775

\begin{tabular}{|c|c|c|c|c|c|c|c|}
\hline $\begin{array}{l}\text { Feature } \\
\text { (1) }\end{array}$ & $\begin{array}{l}V_{\mathrm{sh}}{ }^{a} \\
\mathrm{~km} \mathrm{~s}^{-1} \\
(2)\end{array}$ & $\begin{array}{l}R_{\mathrm{sh}} \\
\mathrm{kpc} \\
(3)\end{array}$ & $\begin{array}{l}D_{\mathrm{sh}} \\
\mathrm{kpc} \\
(4)\end{array}$ & $\begin{array}{l}n_{0} \\
\mathrm{~cm}^{-3} \\
(5)\end{array}$ & $\begin{array}{l}\tau_{\mathrm{sh}, 7}{ }^{b} \\
\text { years } \\
(6)\end{array}$ & $\begin{array}{l}E_{\mathrm{E}, 55}{ }^{c} \\
\text { ergs } \\
(7)\end{array}$ & $\begin{array}{l}E_{\mathrm{c}, 55}{ }^{d} \\
\text { ergs } \\
(8)\end{array}$ \\
\hline $\mathrm{F} 1$ & 54.0 & 2.2 & 4.3 & 0.07 & 4.0 & 1.9 & 0.7 \\
\hline F2 & 62.5 & 2.0 & 5.0 & 0.08 & 3.2 & 2.1 & 0.9 \\
\hline F3 & 75.0 & 1.7 & 7.6 & 0.14 & 2.2 & 3.0 & 2.2 \\
\hline
\end{tabular}

${ }^{a}$ Half of the total velocity width obtained from the PV diagrams in Figs. 8, 9 and 10. See Sect. 3.5.

${ }^{b}$ Kinematical ages in units of $10^{7}$ years.

${ }^{c}$ One-time energy injected in units of $10^{55} \mathrm{ergs}$.

${ }^{d}$ Continuous energy injected in units of $10^{55}$ ergs.

a high global star formation rate, i.e. SFR $\left(M>5 M_{\odot}\right)=$ $2.4 M_{\odot} \mathrm{yr}^{-1}$ (cf. Condon 1992), but creating the features observed requires that $10^{4}$ to $10^{5}$ supernovae occur within the same region on short timescales. These numbers require the presence of 10 to 100 coeval and closely located super star clusters for each supershell, assuming that each contains 1000 supernova-producing stars. We return to this issue in Sect. 4.

\subsubsection{High latitude $\mathrm{H} \alpha$ emission}

Since dust obscuration should be minor at high latitude, the observed high latitude $\mathrm{H} \alpha$ distribution should closely resemble the actual distribution of ionized gas. The largest $\mathrm{H} \alpha$ disk-halo feature occurs on the east side of the NW disk up to $\approx 5.5 \mathrm{kpc}$, in projection, from midplane (Fig. 4a) at the location of F3. Since the correction for inclination is negligible, this is the actual height above the disk, and is much higher than the $\mathrm{H} \alpha$ "worms" found in NGC 891 (Rand et al. 1990) and NGC 4631 (Rand et al. 1992), which reach to about 2 kpc. An unresolved HII region mentioned in Sect. 3.2 at high $z$ $\left(\sim 1.8 \mathrm{kpc}\right.$ in projection) occurs at $\alpha=14^{\mathrm{h}} 53^{\mathrm{m}} 56.5, \delta=$ $3^{\circ} 33^{\prime} 32^{\prime \prime}$, just above the $\mathrm{H} \alpha$ gap. Since the $\mathrm{H} \alpha$ disk is about $30 \mathrm{kpc}$ in diameter and $i=86^{\circ}$, a source at the far edge of the disk will appear at $z=1.0 \mathrm{kpc}$ in projection. Therefore, this source is likely extraplanar and appears to "feed into" the diffuse, large-scale high latitude feature. On the SW disk, there are also two other prominent $\mathrm{H} \alpha$ features at the locations of $\mathrm{F} 1$ and $\mathrm{F} 2$ which are across the major axis from each other (Fig. 4a).

\subsubsection{High latitude radio continuum emission}

Some of the most dramatic features occur in the radio continuum (Fig. 1b) in which extensions can be traced as far as $16 \mathrm{kpc}$, in projection, from the plane. Irwin \& Caron (1994) and Duric et al. (1998) have shown that the extraplanar radio continuum emission is largely non-thermal. The observed extensions show considerable structure far from the plane and may be associated with more distant knots of emission which are disconnected from the disk.
Figures $1 \mathrm{~b}, 2 \mathrm{~b}$ and $2 \mathrm{~d}$ show contours of the $20 \mathrm{~cm}$ continuum data sets at different resolutions and display the growth of features over spatial scales from $5^{\prime \prime}=0.6 \mathrm{kpc}$ to many kpc. Even with these sensitive data, it is not straightforward to associate high resolution features with those observed on larger scales. Cosmic ray diffusion, possibly complex magnetic field geometry, and the fact that several features could be present over any given line of sight in this edge-on galaxy could all be complicating the interpretation of relationships between small and large-scale structure. Nevertheless, some trends can be observed. Generally, if a feature is seen at high resolution, it will also exist farther from the plane at (at least) the next lower resolution.

For example, the several discrete extensions seen on the west side of the NW major axis (Fig. 2d) are observed as smooth extensions at both lower resolutions. Smaller extensions on the west side of the SE major axis are also visible at the lower but not lowest resolutions. Some of this emission at high resolution even appears disconnected from the disk (e.g. $\alpha=14^{\mathrm{h}} 53^{\mathrm{m}} 55^{\mathrm{s}}, \delta=03^{\circ} 31^{\prime} .5$ ) similar to the much larger scale disconnected features seen at low resolution (Fig. 1b).

\subsubsection{High latitude IR emission}

The FIR emission (Fig. 3) shows several features which appear to extend from the disk, most easily seen in the $12 \mu \mathrm{m}$ and $25 \mu \mathrm{m}$ maps due to their higher spatial resolution. While it is not always clear whether an extension is due to noise or a superimposed background source, there are two which have clear counterparts at other wavebands. The first is a small feature extending on the SW side of the disk at $\alpha=14^{\mathrm{h}} 53^{\mathrm{m}} 53.5, \delta=03^{\circ} 31^{\prime} 58^{\prime \prime}$. This feature has a direct HI counterpart (seen in Fig. 6b) and also correlates with extended radio continuum emission (Fig. 2a). The second is on the NW side of the disk at $\alpha=14^{\mathrm{h}} 53^{\mathrm{m}} 58^{\mathrm{s}} .2$, $\delta=03^{\circ} 34^{\prime} 31^{\prime \prime}$. This feature correlates with HI feature F3 and its associated radio continuum and $\mathrm{H} \alpha$ emission. The IR emission occurs at the $3-5 \sigma$ level in each case, but the existence of the features at both $12 \mu \mathrm{m}$ and $25 \mu \mathrm{m}$ and the correlation with the emission at other wavebands argues 
for this emission being real. Weak IR emission at 25 and $60 \mu \mathrm{m}$ can also be seen at the position of one intergalactic radio continuum knot (see $\alpha=14^{\mathrm{h}} 53^{\mathrm{m}} 41.8, \delta=03^{\circ} 31^{\prime} 2^{\prime \prime}$ and Fig. 1b).

While some correlation has been found in the Milky Way Galaxy between IR features, radio continuum features and HII regions (Koo et al. 1992) and also an HI supershell (Kim \& Koo 2000), such correlations have not previously been readily apparent in external galaxies. The FIR features found here are reminiscent of high-latitude FIR in NGC 891 (Alton et al. 1998), M 82, NGC 4631 and NGC 253 (Alton et al. 1999; García-Burillo et al. 2000). In these cases, discrete dusty features are detected out to about $1 \mathrm{kpc}$ in $z$. Extraplanar dust detected optically is also found to be common in a sample of 12 edge-on spiral galaxies by Howk \& Savage (1999). In the Milky Way Galaxy, smaller examples of "dusty worms" are found in Koo et al. (1991). The detection of FIR emission at high- $z$ suggests that the dust is being transported upwards away from the plane with the other components without being destroyed. Various transport mechanisms including radiation pressure and hydrodynamical effects are discussed in Howk \& Savage (1999).

\subsubsection{High latitude $\mathrm{X}$-ray emission}

The X-ray image (Fig. 5) shows 3 main regions in which the emission extends in discrete features, two on the east side of the galaxy and one on the west. As the observations are on a much larger spatial scale than that over which some of the disk-halo features are observed, it is not straightforward to relate the X-ray features directly to other known disk-halo features. However, it is of interest to note that the three X-ray extensions occur roughly at the positions of disk-halo features, F1, F2, and F3. In the case of F3, since this feature can be traced to very large scales in the radio continuum (cf. Fig. 1b), it is possible to associate this disk-halo feature (with more confidence) with the northern X-ray feature on the east side of the galaxy. Since the X-ray resolution is $30^{\prime \prime}$, the highest latitude X-ray emission, if real, is associated with F3 and occurs at $\sim 17 \mathrm{kpc}$, in projection, from the plane, similar to the radio continuum.

\subsection{Relationship between in-disk and extraplanar features}

Figure 7 shows in-disk features (especially $\mathrm{H} \alpha$ ) in comparison with other wavebands. As there are many extraplanar features, the relationship between those in the disk and those above in this edge-on system are not easily established. However, F3, which is seen in every waveband (except for CO for which we have insufficient coverage), is clearly located above a region of low intensity on the major axis between features B and C (as labelled in Fig. 7a). In this region, all sufficiently resolved components of the ISM, except for HI, are at a minimum. There is also evi- dence for extensions away from this feature on the opposite side of the major axis though we have avoided this side due to its proximity to the southern bridge. Thus, for F3, there is an anti-correlation between the high latitude emission and tracers of in-disk star formation, especially in the radio continuum where the intensity dip is not an effect of dust absorption. Extraplanar features F1 and F2 also occur on opposite sides of the SE major axis between A and $\mathrm{B}$ where the in-disk $\mathrm{H} \alpha$ is weaker but the radio continuum is still fairly strong (Sect. 3.2).

\subsection{Correlation of extra-planar features at different wavebands}

Figures 8,9 , and 10 show overlays of all wavebands with sufficient and similar resolutions for the three HI features, F1, F2, and F3. The HI features may appear slightly different from Figs. 1c and d, since they are here averaged only over the velocity range over which they occur.

\subsubsection{F1}

Figure 8 shows a complete HI loop of radius $2.2 \mathrm{kpc}$. The velocity field (Fig. 8b) shows that the bulk of the gas is blueshifted with respect to gas on the major axis. Since this is the receding side of the disk, F1 is lagging behind the general rotation of the galaxy disk. This velocity offset is confirmed from position-velocity slices shown in panels $(\mathrm{g}),(\mathrm{h})$, and $(\mathrm{i})$. These three panels show slices perpendicular to the major axis, corresponding to the left, center, and right positions shown in (a) $(V=0$ represents the systemic velocity). The maximum displacement for "connected" emission between the high latitude gas and gas on the major axis is $\sim 108 \mathrm{~km} \mathrm{~s}^{-1}$, but the velocity offset would be less if $\mathrm{F} 1$ is located at some position other than the projected galactocentric distance. While F1 shows a complete loop structure in position-position space, there is no clear loop structure in position-velocity space, as would be expected if this were an expanding supershell. Rather, there is an interesting double velocity structure evident in the PV slice of Fig. 8i and hinted at in panels (g) and (h). A spur can be seen at $200 \mathrm{~km} \mathrm{~s}^{-1}$ which suggests that redshifted (moving faster than the disk) gas may also be present. This spur appears to extend into some low intensity peaks which move off diagonally towards higher $z$ and higher positive velocity. If this feature is real, then it suggests the presence of gas which is accelerating with $z$ height, with the velocity offset becoming greater than $300 \mathrm{~km} \mathrm{~s}^{-1}$ by $z=50^{\prime \prime}(6 \mathrm{kpc})$. The suggested velocity gradient is $6.75 \mathrm{~km} \mathrm{~s}^{-1} \operatorname{arcsec}^{-1}$ or $56 \mathrm{~km} \mathrm{~s}^{-1} \mathrm{kpc}^{-1}$ up to a height of $z \sim 6 \mathrm{kpc}$. Other faint features can be seen over a wide velocity range at heights up to $140^{\prime \prime}(17 \mathrm{kpc})$ and may form an arc in PV space (center slice). These features exist at the $3 \sigma$ level. 
Figure $8 \mathrm{c}$ shows the $\mathrm{H} \alpha$ emission in greyscale compared with HI contours. The $\mathrm{H} \alpha$ emission reaches high $z$ here and is primarily confined within the HI loop. (Note that the apparent second feature to the NW (right) is due to a stellar artifact. There is only one discrete $\mathrm{H} \alpha$ extension in this figure.) While primarily within the $\mathrm{HI}$ loop, some $\mathrm{H} \alpha$ emission extends to higher $z$ than the HI loop suggesting, along with the PV slices, that the $\mathrm{HI}$ is not closed at the top even though it appears closed in panel (a).

Both the $6 \mathrm{~cm}$ and $20 \mathrm{~cm}$ radio-continuum emission at the position of F1 are extended as seen in Figs. 8d and e, but the emission appears smoother than the HI (even though the resolution is similar) because of cosmic ray diffusion.

The spectral index map shows more structure than either of the radio continuum maps and may prove to be a more useful tracer of activity than the intensity images. For example, the spectral index is steeper within F1 and less steep along the eastern and western limbs of the supershell (Fig. 8f) (see also Collins et al. 2000). Flatter spectral indices can result either from intrinsic flattening of the non-thermal index, such as would occur in shocked regions, or because of a contribution from thermal gas. Since we have the $\mathrm{H} \alpha$ images, it is possible to distinguish between these scenarios. Figure 8c, for example, shows most $\mathrm{H} \alpha$ emission to be within the loop. If the spectral index changes are due to a thermal contribution, then we would expect a flattening within the shell, rather than along the rims, as is observed.

This can be further quantified by estimating how much emission would be required from thermal gas alone to flatten the spectral index by the amount observed $(\sim 0.2)$. We take the non-thermal spectral index, $\alpha_{\mathrm{NT}}$, to be the value within the supershell where the spectral index is steep (i.e. 1.13). Then the required thermal contribution to the flux, $S_{\mathrm{T}}\left(\nu_{1}\right)$, at frequency, $\nu_{1}\left(S_{\nu} \propto \nu^{-\alpha}\right)$ in the ridge is given by

$S_{\mathrm{T}}\left(\nu_{1}\right)=\frac{S_{\mathrm{Tot}}\left(\nu_{1}\right)-\left(\frac{\nu_{1}}{\nu_{2}}\right)^{-\alpha_{\mathrm{NT}}} S_{\mathrm{Tot}}\left(\nu_{2}\right)}{1-\left(\frac{\nu_{1}}{\nu_{2}}\right)^{-\alpha_{\mathrm{NT}}+0.1}}$

where $S_{\text {Tot }}\left(\nu_{i}\right)$ is the measured flux in the ridge at frequency, $\nu_{i}$. For the western ridge, we find that a thermal contribution of $S_{\mathrm{T}}(1.45 \mathrm{GHz})=0.083 \mathrm{mJy}^{\text {beam }^{-1}}$ is required. The electron density, $n_{\mathrm{e}}$, can then be calculated (cf. Mezger \& Henderson 1967) for an adopted geometry. In the simple case of cylindrical geometry, constant density, and an electron temperature of $10^{4} \mathrm{~K}$, this is

$n_{\mathrm{e}}=543 \nu^{0.05} S_{\mathrm{T}}(\nu)^{0.5} \mathcal{D}^{-0.5} \theta^{-1.5} \mathrm{~cm}^{-3}$

where $\mathcal{D}$ is the distance to the source (in $\mathrm{kpc}$ ), $\theta$ is the source diameter $(\operatorname{arcmin})$ and $\nu, S_{\mathrm{T}}(\nu)$ are in $\mathrm{GHz}$ and Jy, respectively. The constant has a weak dependence on frequency, electron temperature and geometry, but varies by no more than $25 \%$ over a wide range of values. Taking $\theta=\theta_{\text {beam }}=0.24$, and the above thermal flux, we find $n_{\mathrm{e}}=0.28 \mathrm{~cm}^{-3}$. The emission measure, $E M$, over a path length, $l$, equal to the beam size, is then $E M=\int n_{\mathrm{e}}^{2} \mathrm{~d} l \approx 132 \mathrm{~cm}^{-6}$ pc. The $\mathrm{H} \alpha$ intensity at $10^{4} \mathrm{~K}$ would be $\int I_{\nu} \mathrm{d} \nu$ and is equivalent to $2.8 \times 10^{-16} \mathrm{erg} \mathrm{s}^{-1} \mathrm{~cm}^{-2} \operatorname{arcsec}^{-2}$. This is an order of magnitude greater than the actual $\mathrm{H} \alpha$ value measured at this position of $1.8 \times 10^{-17} \mathrm{erg} \mathrm{s}^{-1} \mathrm{~cm}^{-2} \operatorname{arcsec}^{-2}$ and thus a greater thermal contribution than is observed is required to flatten the spectral index.

We conclude that the spectral index flattening is not due to a thermal contribution, but rather is due to real differences in the non-thermal spectral index. This conclusion does not change if we make reasonable alterations to the geometry or positions chosen for measurement. Given that the radio continuum brightness in the ridges is also greater, the spectral index has probably been flattened in shocked regions. Rand (2000) has suggested that the optical line ratios in high latitude gas cannot be explained by photoionization alone, but require either a changing temperature or a contribution from shocks. These results are consistent with a shock explanation.

\subsubsection{F2}

The second feature, F2, is on the SE major axis, extending towards the west (Fig. 1d) and is shown in Fig. 9. As with F1, this feature has a loop-like appearance with an additional feature extending to the west. The velocity field of this supershell (Fig. 9b) is also slightly blueshifted with respect to the disk below. Since this part of the disk is receding from the observer, the bulk of the emission from F2 is lagging with respect to disk rotation. Slices perpendicular to the major axis, shown in panels (g), (h), and (i), confirm this result but also reveal a more complex structure. The center slice clearly reveals the signature of an expanding shell in PV space with a full width of $125 \mathrm{~km} \mathrm{~s}^{-1}$. This feature appears to have a closed top. The left and right slices, however, both show a double structure, similar to that seen in F1 but more obviously. The left slice shows a redshifted spur in the direction of a disconnected feature which again, suggests acceleration with $z$ height. In this case, the gradient is $5.0 \mathrm{~km} \mathrm{~s}^{-1} \operatorname{arcsec}^{-1}$ or $41 \mathrm{~km} \mathrm{~s}^{-1} \mathrm{kpc}^{-1}$ to a height of $45^{\prime \prime}$ or $5.4 \mathrm{kpc}$. The right slice shows a clear split profile with a velocity separation of $\sim 165 \mathrm{~km} \mathrm{~s}^{-1}$ and no evidence for acceleration. These features have open tops. It is possible that we are observing the superposition of 3 different features along this line of sight. However, the loop-like appearance of the feature in position-position space, the loop-like spectral index distribution (see below), the location of F2 across the major axis with respect to F1, and the absence of similar striking features elsewhere (aside from F3) suggests that we are mainly observing a single feature.

As in $\mathrm{F} 1$, the $\mathrm{H} \alpha$ emission (Fig. 9c) reaches high latitude and occurs mostly within the supershell. At the centre of the supershell where it is connected to the disk, an obvious $\mathrm{H} \alpha$ "worm" can be seen amidst other smaller discrete vertical features. 

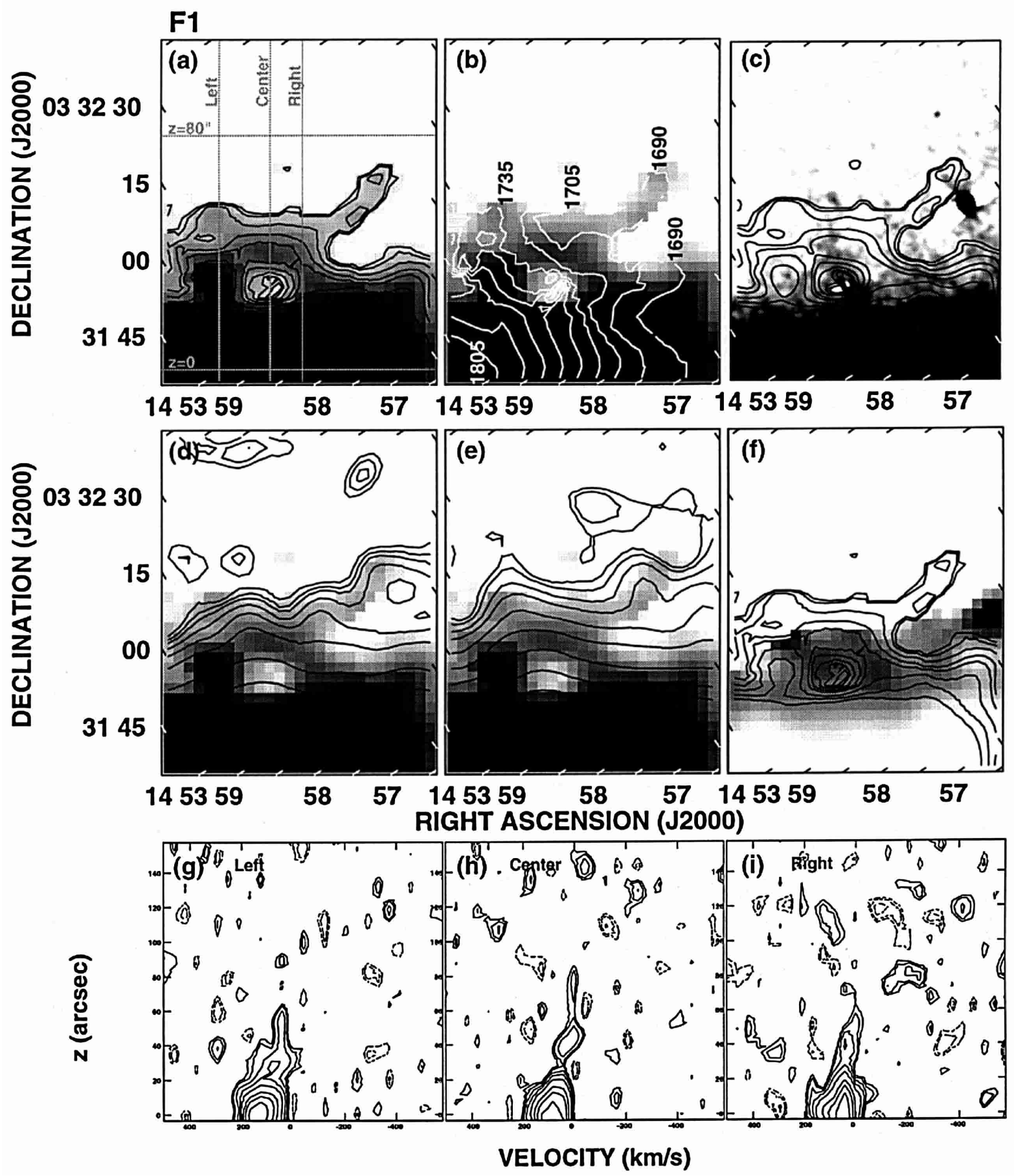

Fig. 8. The HI loop, F1, shown in comparison to data at other wavebands. In each frame, the HI has been integrated over velocities, 1683.4 to $1850.2 \mathrm{~km} \mathrm{~s}^{-1}$ and the vertical extent of each frame is $119^{\prime \prime}$. a) Integrated intensity HI. The greyscale ranges from 15 (white) to 200 (black) mJy beam ${ }^{-1} \mathrm{~km} \mathrm{~s}^{-1}$ and contours are at 40, 50, 72, 100, 125, and $175 \mathrm{mJy} \mathrm{beam}^{-1} \mathrm{~km} \mathrm{~s}^{-1}$, and so throughout unless otherwise indicated. b) Intensity-weighted mean velocities over HI (here, the greyscale peak flux is $180 \mathrm{mJy} \mathrm{beam}^{-1}$ ). Velocity contours are separated by $15 \mathrm{~km} \mathrm{~s}^{-1}$. c) HI over $\mathrm{H} \alpha$. The greyscale ranges from $16 \times 10^{-19} \mathrm{ergs} \mathrm{s}^{-1} \mathrm{~cm}^{-2} \mathrm{pixel}^{-1}$ (white, $2 \sigma$ ) to $64 \times 10^{-19} \mathrm{ergs} \mathrm{s}^{-1} \mathrm{~cm}^{-2}$ pixel $^{-1}$ (black). Two residual star images are visible in the H $\alpha$ image, one at the center of the HI loop and one to the upper right. d) $6 \mathrm{~cm}$ radio continuum over HI. Contours are 0.02, 0.03, 0.04, 0.06, 0.09, 0.15, 0.35 and $0.75 \mathrm{mJy}_{\text {beam }}{ }^{-1}$. e) $20 \mathrm{~cm}$ radio continuum over HI. Contours are $0.054,0.075,0.12,0.18,0.30,0.50,1.0,2.0$, and $3.5 \mathrm{mJy}_{\text {beam }}{ }^{-1}$. f) HI over spectral index map. The greyscale ranges from 0.8 (white) to 1.2 (black). g) $\rightarrow \mathbf{i}$ ) Position-velocity slices through the HI cube, taken along vertical lines denoted Left, Center, and Right in a). Contours are at \pm 0.6 (1.5 $\sigma), \pm 0.8$, $\pm 1.2,1.8,2.8,5.0,7.0$, and $10 \mathrm{mJy}_{\text {beam }}{ }^{-1}$. 

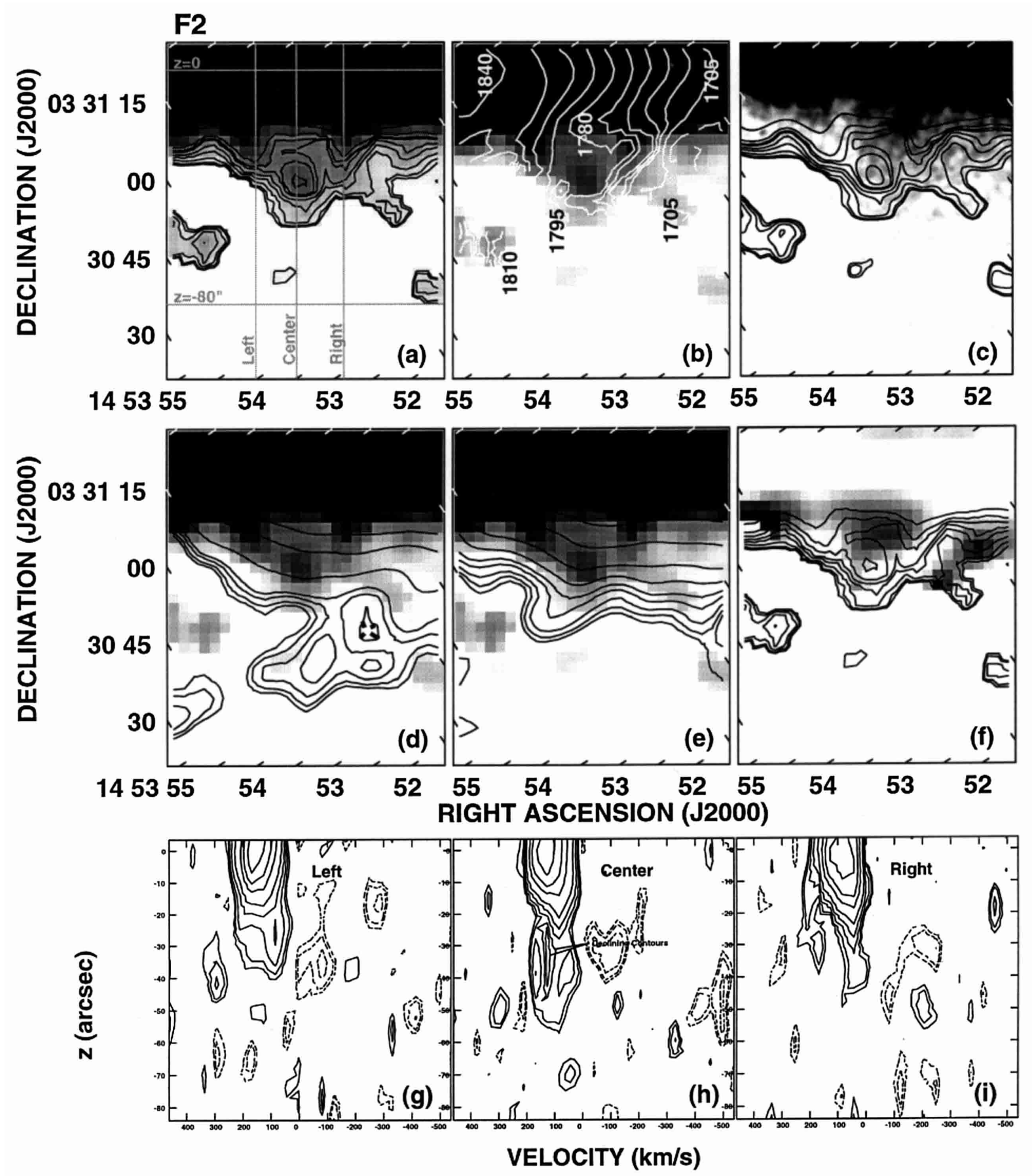

Fig. 9. The HI loop, F2, shown in comparison to data at other wavebands. In each frame, the HI has been integrated over velocities, 1683.4 to $1892.0 \mathrm{~km} \mathrm{~s}^{-1}$ and the vertical extent of each frame is $147^{\prime \prime}$. a) Integrated intensity HI. The greyscale ranges from 15 (white) to 300 (black) mJybeam ${ }^{-1} \mathrm{~km} \mathrm{~s}^{-1}$ and contours are at 40, 50, 70, 100, 126, 156, 170, 200, and $300 \mathrm{mJy}_{\text {beam }}{ }^{-1} \mathrm{~km} \mathrm{~s}^{-1}$, and so throughout, unless otherwise indicated. b) Intensity-weighted mean velocities over HI (here, the greyscale peak is $250 \mathrm{mJy}$ beam ${ }^{-1} \mathrm{~km} \mathrm{~s}^{-1}$ ). Velocity contours are separated by $15 \mathrm{~km} \mathrm{~s}^{-1}$. c) HI over H $\alpha$. The greyscale ranges from $16 \times 10^{-19} \mathrm{ergs} \mathrm{s}^{-1} \mathrm{~cm}^{-2}$ pixel $^{-1}$ (white, $2 \sigma$ ) to $80 \times 10^{-19} \mathrm{ergs} \mathrm{s}^{-1} \mathrm{~cm}^{-2}$ pixel $^{-1}$ (black). d) $6 \mathrm{~cm}$ radio continuum over

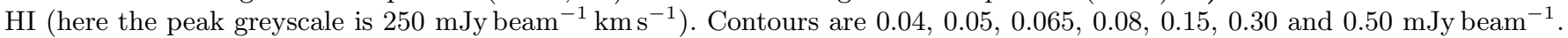
The hatched region indicates declining contours. e) $20 \mathrm{~cm}$ radio continuum over HI (peak greyscale is $250 \mathrm{mJy} \mathrm{beam}^{-1} \mathrm{~km} \mathrm{~s}^{-1}$ ). Contours are $0.05,0.07,0.10,0.15,0.20,0.30,0.50,1.0$, and $2.0 \mathrm{mJy}$ beam $^{-1}$. f) HI over spectral index map. The greyscale ranges from 0.8 (white) to 1.0 (black). g) $\rightarrow$ i) Position-velocity slices through the HI cube, taken along vertical lines denoted Left, Center, and Right in a). Contours are at $\pm 0.6(1.5 \sigma), \pm 0.8, \pm 1.2,1.8,2.8,5.0,7.0$, and $10 \mathrm{mJy} \mathrm{beam}^{-1}$. 
The $6 \mathrm{~cm}$ contours in Fig. 9d show an inverted $T$-shaped plume extending from one side of $\mathrm{F} 2$ and almost connects with an extension west of it. This feature, and some others, bears an interesting resemblance to those seen on the sun. In contrast, the $20 \mathrm{~cm}$ contours show a single discrete extension centered on F2 and another one which corresponds to the $6 \mathrm{~cm}$ extension in the western edge of the frame. The spectral indices (Fig. 9f) steepen within $\mathrm{F} 2$ coincident with the $\mathrm{H} \alpha$ worm. As in F1, the eastern and western limbs of F2 as well as the top show a striking flattening of the spectral index. A similar analysis to F1 indicates that, again, this flattening must be intrinsic to the non-thermal spectrum, suggesting the presence of shocks.

\subsubsection{F3}

The disturbance related to F3 (Fig. 10) is the largest and most obvious in this galaxy. The HI map shows the supershell to be open-topped, unlike the projected appearance of F1 and (to some extent) F2. The velocity field shown in Fig. 10b is chaotic but is generally redshifted with respect to the disk gas. Since the underlying disk is advancing, the bulk of the gas lags behind the disk. This is again illustrated in the PV slices shown in panels $(\mathrm{g}),(\mathrm{h})$, and (i). There is only a hint, in panel (i), of double structure showing some blueshifted gas. If the blueshifted feature is real, then the velocity separation is $\sim 150 \mathrm{~km} \mathrm{~s}^{-1}$. All of the slices show open tops and also show many smaller features over a wide velocity range at $z \sim 60^{\prime \prime}(7.2 \mathrm{kpc})$. It is of interest to examine the left slice, especially, since this is roughly where, in optical emission, Rand (2000) has measured a steady decelerating velocity gradient of $\sim 22 \mathrm{~km} \mathrm{~s}^{-1} \mathrm{kpc}^{-1}$ with $z$ until $z \sim 5 \mathrm{kpc}$ after which the velocity remains roughly constant at $1660 \mathrm{~km} \mathrm{~s}^{-1}$, or $-20 \mathrm{~km} \mathrm{~s}^{-1}$ with respect to systemic (Slit 1 in Rand 2000). From Fig. 10g, we find similar behaviour. A curve through the brightest emission shows decreasing velocities until $z \sim 40^{\prime \prime}(5 \mathrm{kpc})$ and then remains constant near $V_{\text {sys }}$. The velocity gradient is $\sim 17 \mathrm{~km} \mathrm{~s}^{-1} \mathrm{kpc}^{-1}$ which we take to be consistent with Rand's value, given the fact that this gradient shows some curvature, and the fact that the resolutions of the two data sets are quite different. Note also that Fig. $8 \mathrm{~g}$, through F1, shows a similar trend as Rand's Slit 2 (HI velocity gradient of about $17 \mathrm{~km} \mathrm{~s}^{-1} \mathrm{kpc}^{-1}$ versus Rand's $21 \mathrm{~km} \mathrm{~s}^{-1} \mathrm{kpc}^{-1}$ ), although Slit 2 is offset by about $22^{\prime \prime}$ from F1.

In Fig. 10c, the $\mathrm{H} \alpha$ emission again fills the inside edge of the supershell but, in this case, the $\mathrm{H} \alpha$ emission clearly shows its own double open-topped structure and occurs on the inner rim of the HI features. This, along with the similarity in $\mathrm{H} \alpha / \mathrm{HI}$ velocity structure along the left slice suggests that the $\mathrm{H} \alpha$ emission represents the inner ionized skin of the HI supershells.

Correlations between the $6 \mathrm{~cm}, 20 \mathrm{~cm}$ emission and $\mathrm{HI}$ are difficult to discern because the radio continuum emission displays many extensions in this region. It is noteworthy that the radio continuum emission shows disconnected pieces at high latitude, reminiscent of the inverted $T$-shaped piece in $\mathrm{F} 2$ after being detached from the galaxy. For F3, the spectral index does not show as clear a signature as the other two features, although a ridge of flatter spectrum emission can be seen in a feature to the left.

\subsubsection{Higher latitude emission}

It is very interesting that, for F1, F2, and F3, there appears to be a change of character in the disk-halo features at $z$ heights of between $\sim 50$ and $60^{\prime \prime}$ or $\sim 6$ to $7 \mathrm{kpc}$. At this height, the features appear to break up into many smaller features which, individually, are at low $\mathrm{S} / \mathrm{N}$, but form coherent enough structures in PV space that they appear to be real. For example, related to F1, a curved string of emission features is seen at heights up to $z \sim 140^{\prime \prime}$ or $17 \mathrm{kpc}$ (Fig. 8h) which is roughly the maximum height observed in any component related to this galaxy. F3 (Fig. 10h) also shows a string of HI features reaching $z \sim 65^{\prime \prime}$, or $8 \mathrm{kpc}$. Similar PV features have also been observed in the galaxy, NGC 2613, above high latitude HI extensions (Chaves \& Irwin 2001).

These features occur over a very wide range of velocities, both positive and negative with respect to galaxy rotation. This is consistent with the implication from the $\mathrm{HI}$ velocities that both redshifted and blueshifted gas may be present, even though the lagging gas dominates at low latitudes. Although we have avoided the region on the other side of the major axis from feature F3 due to its proximity to the southern bridge, it is worth showing, in Fig. 11 a single PV slice at the same position as Fig. 10h but extending to the south of the major axis as well. The individual features occur on both sides of the major axis and at almost all velocities. The impression is that, around $z$ of $5-7 \mathrm{kpc}$, the feature has become unstable and broken up into many cloudlets which exist over a wide range of velocities more typical of Population II objects.

\subsection{Anatomy of an HI supershell}

Based on the above results, we have put together a picture of a "typical" HI supershell in NGC 5775. This is shown in Fig. 12. The particular display is most relevant to feature, F2, but the general appearance will be similar for the others, though the presence and extent of the cap and sides will vary. The inner rims of the HI walls will be ionized and shocks will also exist within the walls, flattening the spectral index there. Hot, X-ray emitting gas will also be present. In this picture, the feature is formed by an outflowing conical wind and the HI accelerates in both the redshifted and blueshifted direction with respect to underlying rotation. At higher $z$, the lagging side of the feature dominates and a coherent structure is less obvious as the supershell breaks up. At the highest $z$, the feature may be partially or fully open and smaller clouds of $\mathrm{HI}$ continue to be present. 
F3
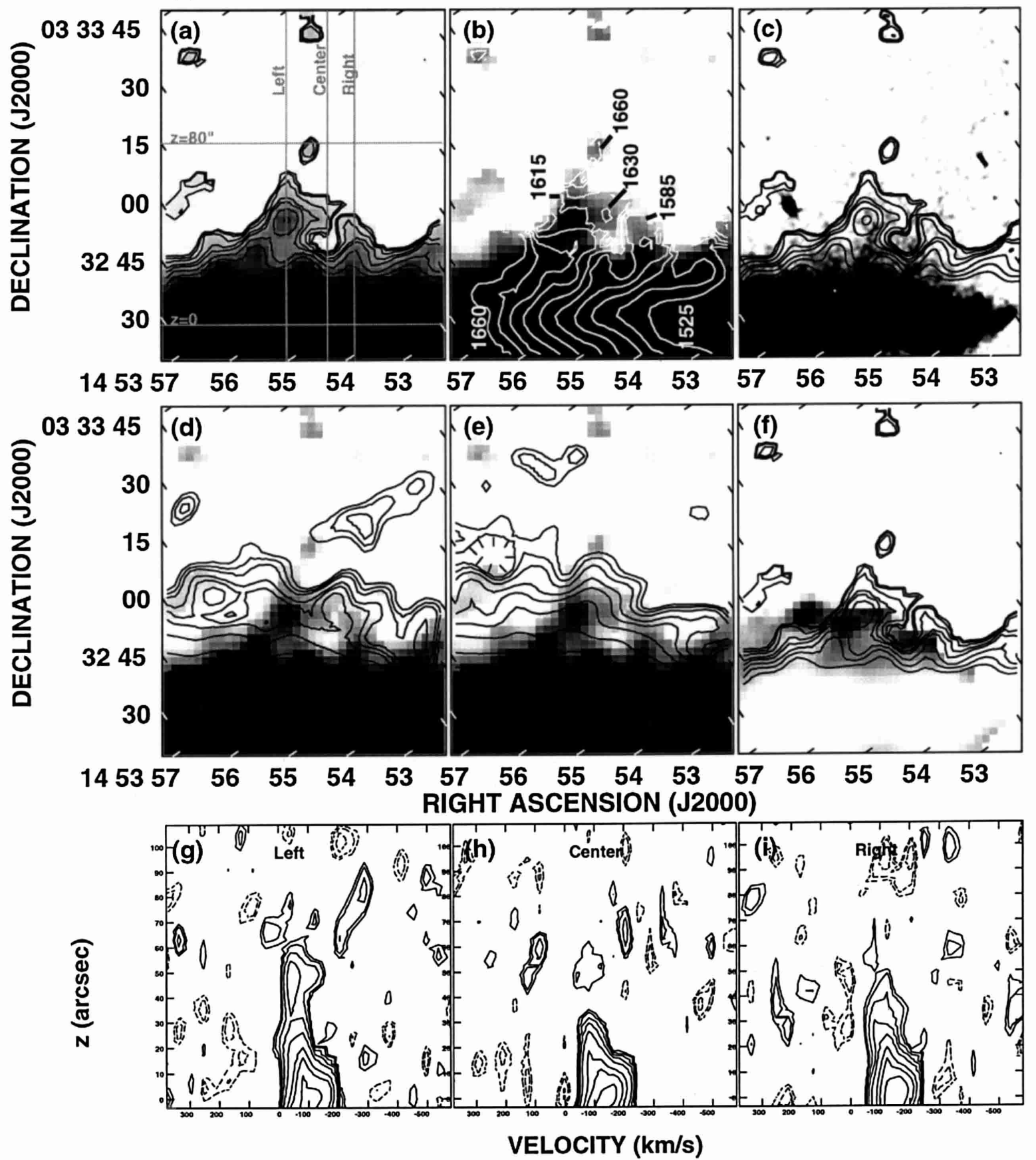

Fig. 10. The HI loop, F3, shown in comparison to data at other wavebands. In each frame, the HI has been integrated over velocities, 1475.0 to $1683.4 \mathrm{~km} \mathrm{~s}^{-1}$ and the vertical extent of each frame is $161^{\prime \prime}$. a) Integrated intensity HI. The greyscale ranges from 15 (white) to 300 (black) mJybeam ${ }^{-1} \mathrm{~km} \mathrm{~s}^{-1}$ and contours are at 40, 50, 70, 100, 125, 175, and $230 \mathrm{mJy}_{\mathrm{beam}}^{-1} \mathrm{~km} \mathrm{~s}^{-1}$, and so throughout, unless otherwise indicated. b) Intensity-weighted mean velocities over HI (here, the peak greyscale is 150 mJy beam $\left.{ }^{-1}\right)$. Velocity contours are separated by $15 \mathrm{~km} \mathrm{~s}^{-1}$. c) HI over H $\alpha$. The greyscale ranges from $28 \times 10^{-19} \mathrm{ergs} \mathrm{s}^{-1} \mathrm{~cm}^{-2}$ pixel $^{-1}$ (white, $3.5 \sigma$ ) to $64 \times 10^{-19} \mathrm{ergs} \mathrm{s}^{-1} \mathrm{~cm}^{-2}$ pixel $^{-1}$ (black). Two residual star images are visible in the $\mathrm{H} \alpha$ image, one to the upper left of the $\mathrm{HI}$ loop and one to the upper right. d) $6 \mathrm{~cm}$ radio continuum over HI (here, the peak greyscale is set to $200 \mathrm{mJy} \mathrm{beam}^{-1} \mathrm{~km} \mathrm{~s}^{-1}$ ). Contours are $0.02,0.03,0.04,0.06,0.072,0.09,0.15$, and $0.30 \mathrm{mJy} \mathrm{beam}^{-1}$. e) $20 \mathrm{~cm}$ radio continuum over HI total intensity. Contours are $0.050,0.070,0.10,0.15,0.20,0.30,0.50$, and $^{1.0} \mathrm{mJy} \mathrm{beam}^{-1}$. f) HI over spectral index map. The greyscale ranges from 0.85 (white) to 1.3 (black). g) $\rightarrow$ i) Position-velocity slices through the HI cube, taken along vertical lines denoted Left, Center, and Right in a). Contours are at $\pm 0.6(1.5 \sigma), \pm 0.8, \pm 1.2,1.8,2.8$, 5.0, 7.0, and $10 \mathrm{mJy}_{\text {beam }}{ }^{-1}$. 


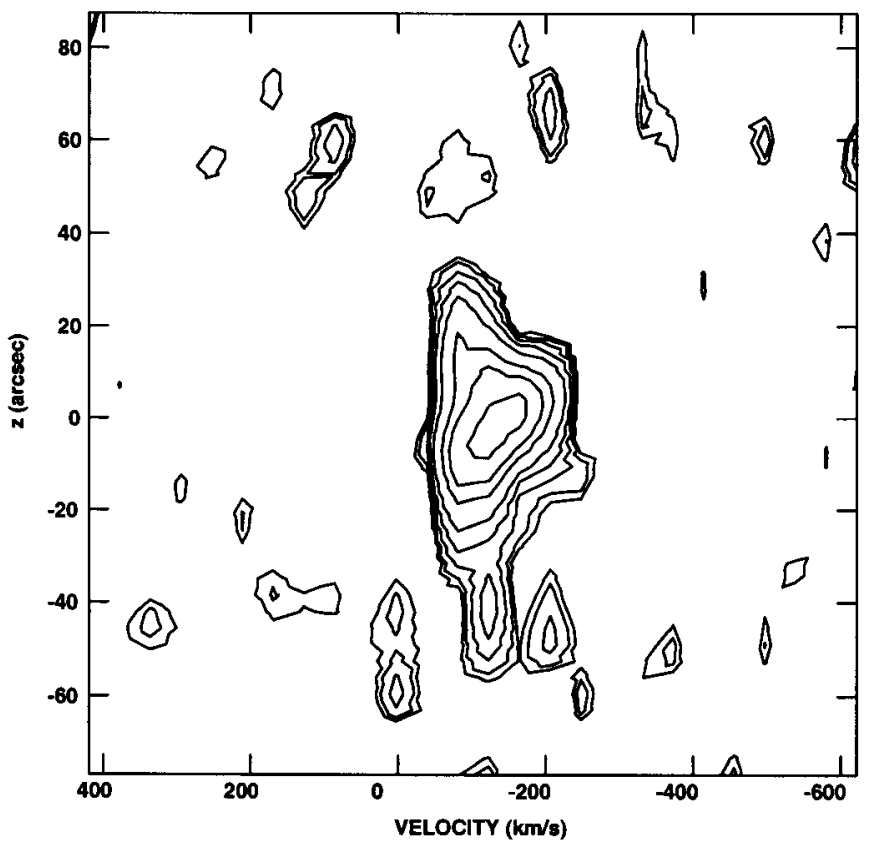

Fig. 11. Vertical position-velocity slice through the center of the HI loop, F3, at the same position as in Fig. 10h, but including the region below the major axis.

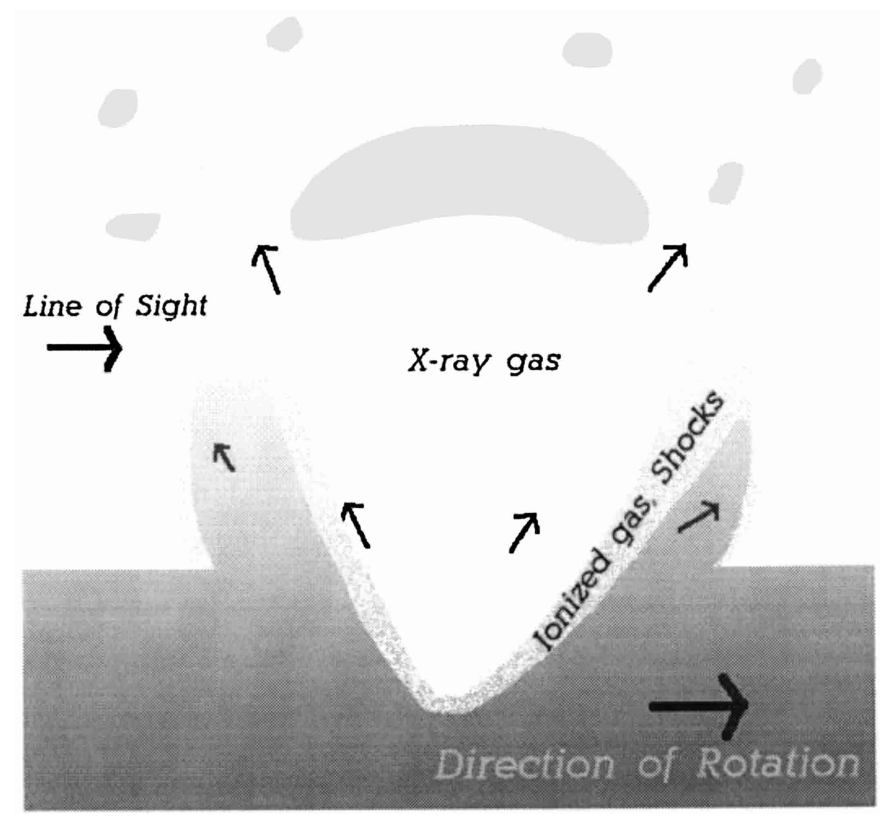

Fig. 12. Sketch of an HI supershell, as typified by the features, F1, F2, and F3 in NGC 5775.

\section{Discussion}

Our multi-wavelength approach has proven to be fruitful and, in fact, necessary in order to understand the activity in this galaxy. Information on each component of the ISM has allowed us to piece together a view of the diskhalo interaction with unprecedented detail. Some important observations are that a) all components of the ISM (with the exception of $\mathrm{CO}$ for which there is insufficient spatial coverage) are represented in a disk-halo feature, b) there is a minimum in the emission suggesting a lower level of star formation in the disk under the largest feature, F3, c) the bulk of the HI in discrete extensions tends to lag with respect to the underlying disk and $\mathrm{d}$ ) the velocities are suggestive that gas is accelerating with higher $z$ and clearly show the presence of an expanding supershell in the case of F2. These observations suggest that we are dealing with outflows and rule out inflows for the observed features. We seem to be viewing the remnants of supershells as they expand and break up through the disk-halo interface.

Based on the features seen in NGC 5775, we propose a generic disk-halo structure such as is illustrated in Fig. 12. In this picture, gas accelerates as it ascends into the halo, possibly from cone-like outflow. The split line profiles or expansion signatures in PV space indicate a classical supershell structure. However, the features are either opentopped or only partially capped and ionized gas, as seen in $\mathrm{H} \alpha$ emission, escapes out of the top. The ionized gas also forms an inner skin on the HI as shown spatially as well as in velocity structure which is consistent with the HI. The existence and extent of the cap may vary. Thus, the signature of a complete "supershell" may or may not be present. Both red and blueshifted gas are present in these features as well as evidence for acceleration away from the plane. However, it is the lagging gas which dominates. The possibility of a globally lagging halo will be investigated in a separate paper. At higher $z$ there appears to be a transition region between 5 and $7 \mathrm{kpc}$ in which the shell breaks up and HI clumps are seen over a wide range of velocities.

Let us now review the possible formation mechanisms and consider which, if any, may be applicable to NGC 5775.

\subsection{Supernovae}

The effect of supernova explosions on the ISM has been widely studied both observationally in our own and other galaxies (e.g., Katgert 1969; Heiles 1979; Westerlund \& Mathewson 1966; Brinks \& Bajaja 1986) and theoretically (Bruhweiler et al. 1980; Tomisaka et al. 1981; Mac Low \& McCray 1988). Galactic "fountain" and "chimney" models (Shapiro \& Field 1976; Norman \& Ikeuchi 1989) suggest that supershells are produced from multiple supernovae, both temporally and spatially correlated. These shells may then break out of the disk and provide channels through which hot gas can escape into the halo.

In the case of NGC 5775, the high mass star formation rate (SFR) inferred by the FIR luminosity $\left(2.6 \times 10^{10} L_{\odot}\right.$, using the equation given in Lonsdale Persson \& Helou 1987) is $2.4 M_{\odot} \mathrm{yr}^{-1}$ (from Eq. (26) in Condon 1992). This is comparable to the SFR in M 82 (Young \& Scoville 1984). It is therefore conceivable that massive stars play an important role in shaping the ISM in this galaxy. Kennicutt et al. (1989) find 
that the brightest HII region in normal late-type galaxies contains the equivalent of about $20000 \mathrm{O}$ and B stars, though very few galaxies have such large associations. Over a hundred super star clusters (SSCs) have also been discovered in the starburst nucleus of M 82 using the Hubble Space Telescope. Some of these are extremely luminous $\left(M_{V} \sim-16^{\mathrm{m}}\right)$ and massive $\left(\sim 10^{7} M_{\odot}\right)$ with ages comparable to the ages of the supershells in NGC 5775 (O'Connell et al. 1995; Gallagher \& Smith 1999). While we do not have information on the SSC population in NGC 5775, the limitations of supernovae alone as an explanation for the observed supershells are that a) a very large number $\left(10^{4}\right.$ to $\left.10^{5}\right)$ of correlated supernovae are required, and b) there is little evidence for such activity at the base of feature F3. If activity related to massive star formation has indeed formed F3, then it must have declined rapidly within the lifetime of the feature $\left(\sim 2.2 \times 10^{7}\right.$ yrs $)$.

Several recent studies have shown that when magnetic fields are included in numerical simulations not just as a secondary effect, then large shells and blow-out can occur with more modest energy requirements (e.g., Kamaya et al. 1996). In the process, the field lines drag the partially ionized gas, dust and energetic cosmic rays along to high latitude. Sofue et al. (1994) find that this "magnetic flux inflation" can be used to explain the multitude of out-of-plane dust features in NGC 253. Thus, magnetic instabilities could exist as a non-negligible enhancement to the primary supernova mechanism for producing HI supershells.

\subsection{Hypernovae}

Another possible energy source for supershell formation, which alleviates the requirement of extraordinarily large clusters, is hypernovae (see also Efremov et al. 1998; Paczyński 1998) which may be able to release over $10^{54}$ ergs of kinetic energy and have been proposed as a possible progenitor of gamma-ray bursters (GRBs). The frequency of GRBs, assuming they are powered by hypernovae, is $10^{4}-10^{5}$ times less common than supernovae (Paczyński 1998). We estimate the supernova rate from Condon (1992), $\nu_{\mathrm{SN}}=3.7 \times 10^{-12} L_{\mathrm{FIR}}=0.1 \mathrm{yr}^{-1}$, leading to a hypernova rate of about $10^{-5}-10^{-6} \mathrm{yr}^{-1}$. We therefore expect to see 10-100 hypernovae within a period of $10^{7}$ years or the lifetime of the HI features. Since 6 HI supershells have been identified in Lee (1998), this is roughly consistent with the proposed hypernova rate, though it should be kept in mind that the characteristics of such objects, if they exist, are not well known.

\subsection{External cloud impacts}

The impact of high velocity clouds (HVCs) with the disk has been proposed as the formation mechanism of some HI supershells in our own Galaxy (Mirabel 1982; Meyerdierks 1991) and in external galaxies in which input energies are large. While we have argued (Sect. 3.5) that the features seen in NGC 5775 represent outflows, it is interesting to note that outflow features are also predicted in the infalling cloud scenario, for example, an impact with a magnetized disk can produce outflowing velocities $>100 \mathrm{~km} \mathrm{~s}^{-1}$ and acceleration with $z$ height (Santillán et al. 1999).

Since NGC 5775 is clearly interacting with at least NGC 5774, a source of clouds is potentially present. To help determine whether impacting clouds can explain the outflow on energetic grounds, we adopt an impacting cloud mass of $2 \times 10^{7} M_{\odot}$, similar to that found by Rand \& Stone (1996), and a $z$ height of $35 \mathrm{kpc}$ as a typical initial position (corresponding to the HI emission feature seen in Fig. $1 \mathrm{~d}$ at $\left.\alpha=14^{\mathrm{h}} 53^{\mathrm{m}} 40^{\mathrm{s}}, \delta=3^{\circ} 30^{\prime} 30^{\prime \prime}\right)$. The potential energy of such a cloud is (Binney \& Tremaine 1987)

$$
\begin{gathered}
\Phi(z)=2 \times 10^{54}\left(\frac{M_{\text {cloud }}}{2 \times 10^{7} M_{\odot}}\right)\left(\frac{z_{\mathrm{o}}}{700 \mathrm{pc}}\right)^{2} . \\
\left(\frac{\rho_{\mathrm{o}}}{0.185 M_{\odot} \mathrm{pc}^{-3}}\right) \ln \left[\cosh \frac{z}{z_{\mathrm{o}}}\right],
\end{gathered}
$$

where $z_{\mathrm{o}}$ is the scale height of the vertical distribution of stellar mass and $\rho_{\mathrm{o}}$ is the stellar mass density at the midplane. The last two parameters take the Galactic and solar neighbourhood values, respectively. We can then equate the kinetic energy at the point when the cloud hit the galactic plane $\left(\frac{1}{2} M_{\text {cloud }} v^{2}\right.$, where $v$ is the velocity at the point of contact) with the potential energy to obtain the velocity of impact. This gives $v=66 \mathrm{~km} \mathrm{~s}^{-1}$. Santillán et al. (1999) have shown that the resulting outflowing gas velocities after impact can achieve $64 \%$ to $100 \%$ of the infalling cloud velocity. Thus, this value is consistent with the expansion velocities of the supershells observed here. Consequently, on energetic grounds, impacting clouds could be responsible for the observed features.

However, for NGC 5775, this model has more difficulty accounting for the velocity structure of the features and their symmetry. For example, in the models of Santillán et al., outflows are produced when clouds "rebound" from the disk via magnetic tension. Thus, the cloud is not expected to penetrate the disk and outflows would not be seen on both sides of the disk (as with F1, F2) unless there happens to be an infalling cloud symmetrically on the other side of the disk as well. In any case, one would not expect to see closed loops in projection (e.g., F1) from such "splashback" and one would not expect to see the signature of an expanding shell in PV space (e.g., F2). If an impacting cloud somehow passed through the disk, again, it is unlikely that there would be evidence for outflow on both sides. Finally, high velocity cloud impacts with a velocity less than $300 \mathrm{~km} \mathrm{~s}^{-1}$ cannot shock-heat gas into $\mathrm{X}$-ray-emitting temperature. If the X-ray emission from the high- $z$ features is real, we can exclude this mechanism. Thus, even though NGC 5775 is an interacting galaxy, the anatomy of the disk-halo features in NGC 5775 is inconsistent with the impacting cloud scenario. 


\section{Summary and conclusions}

In this paper, we examined the distribution of the various ISM components in the edge-on, infrared-bright, interacting galaxy, NGC 5775 which shows an active disk-halo interface. All components of the ISM are presented, including emission from $\mathrm{HI}$, the radio continuum, CO, FIR, $\mathrm{X}$-ray and $\mathrm{H} \alpha$. NGC 5775 contains at least 3 well-defined HI disk-halo features which we have interpreted as representing supershells or remnants of supershells as they evolve through the disk-halo interface.

NGC 5775 is also interacting with its companion galaxy, NGC 5774, (and possibly IC 1070 as well) and this is manifested in the form of two bridges connecting the two galaxies, as well as a possible tidal tail at the southeastern tip of NGC 5775. In particular, the southern intergalactic bridge can be traced in all observed wavebands except $\mathrm{CO}$ for which data are not yet available.

At least three obvious extraplanar features (F1, F2 and F3) can be traced in HI. The largest of these (F3) reaches a projected height of $7.3 \mathrm{kpc}$. The energy required to produce each of these features is high, of order $10^{55} \mathrm{ergs}$, or equivalent to $10^{4}$ supernovae. High latitude emission associated with such features can be seen in all wavebands, representing all components of the ISM (except CO for which there is insufficient coverage). This includes the FIR emission, indicating the presence of dust at high latitudes as well. There is a minimum in the disk emission beneath the most obvious disk-halo feature, F3. Evidence for acceleration away from the plane and double line velocity structure with both red and blueshifted sides are seen. One feature (F2) shows the clear signature of an expanding supershell in $\mathrm{PV}$ space. The others do not, but are suggestive of parts of a supershell which have broken up.

$\mathrm{H} \alpha$ emission is located interior to the $\mathrm{HI}$ and exhibits similar velocity structure where measured (F3). From the radio continuum data, we have clearly shown that the flattening of the spectral indices at the ridges of the features are due to shocks and cannot be attributed to a contribution from thermal gas.

Based on the above results, a picture of a typical HI supershell is presented where the supershell is created by an outflowing conical wind. X-ray emitting gas and ionized gas exist within the HI supershell. At high- $z$, the parts of the features that are lagging with respect to the galactic rotation dominate (see Fig. 12). At higher $z$, numerous smaller clumps at both red and blueshifted velocities are seen.

Although NGC 5775 is interacting, the symmetry and velocity structure of the observed disk-halo features are inconsistent with the impacting cloud scenario. These features are likely internally generated via a violent process, given the energies and shocks observed. If supernovae are the origin of the features, then of order $10^{4}$ correlated supernovae are required and must fade within a time period which is shorter than the lifetime of the features.

Note added in proof: Since the original manuscript of this paper was written, we have made clear detections of
CO (1-0) and CO (2-1) in F2 and also $850 \mu \mathrm{m}$ dust emission in F1, F2, and F3.

Acknowledgements. S. W. L. would like to thank Dr. D. Wing for the many useful discussions related to this paper. J. I. wishes to thank the Natural Sciences and Engineering Research Council of Canada for a research grant. This work has made use of the Digitized Sky Survey of the Space Telescope Science Institute. The Space Telescope Science Institute is operated by the Association of Universities for Research in Astronomy, Inc. for the National Aeronautics and Space Administration. The Digitized Sky Survey was produced under Government grant NAG W-2166. We have also made use of the NASA/IPAC Extragalactic Database (NED) which is operated by the Jet Propulsion Laboratory, under contract with the National Aeronautics and Space Administration.

\section{References}

Alton, P. B., Bianchi, S., Rand, R. J., et al. 1998, ApJL, 507, L125

Alton, P. B., Davies, J. I., \& Bianchi, S. 1999, A\&A, 343, 51

Aumann, H. H., Fowler, J. W., \& Melnyk, M. 1990, AJ, 99, 1674

Binney, J., \& Tremaine, S. 1987, Galactic Dynamics (Princeton, New Jersey: Princeton University Press)

Bregman, J. N., \& Pildis, R. A. 1994, ApJ, 420, 570

Brinks, E., \& Bajaja, E. 1986, A\&A, 169, 14

Bruhweiler, F. C., Gull, T. R., Kafatos, M., \& Sofia, S. 1980, ApJL, 238, L27

Chaves, T. A., \& Irwin, J. A. 2001, ApJ, 557, 646

Chevalier, R. A. 1974, ApJ, 188, 501

Collins, J. A., Rand, R. J., Duric, N., \& Walterbos, R. A. M. 2000, ApJ, 536, 645

Condon, J. J. 1992, ARA\&A, 30, 575

Dahlem, M., Heckman, T. M., Fabbiano, G., Lehnert, M. D., \& Gilmore, D. 1996, ApJ, 461, 724

Dettmar, R.-J. 1992, Fundam. Cosmic Phys., 15, 143

Duric, N., Irwin, J. A., \& Bloemen, H. 1998, A\&A, 331, 428

Efremov, Y. N., Elmegreen, B. G., \& Hodge, P. W. 1998, ApJ, 501, L163

Gallagher, J. S., \& Smith, L. J. 1999, MNRAS, 304, 540

García-Burillo, S., Martín-Pintado, J., Fuente, A., \& Neri, R. 2000, A\&A, 355, 499

Heiles, C. 1979, ApJ, 229, 533

Heiles, C. 1984, ApJS, 55, 585

Howk, J. C., \& Savage, B. D. 1999, AJ, 117, 2077

Hummel, E., Beck, R., \& Dettmar, R.-J. 1991, A\&AS, 87, 309

Irwin, J. A. 1994, ApJ, 429, 618

Irwin, J. A., \& Caron, B. L. 1994, in Mass-Transfer Induced Activity in Galaxies, ed. I. Shlosman (Cambridge University Press, Cambridge), 362

Irwin, J. A., \& Seaquist, E. R. 1990, ApJ, 353, 469

Irwin, J. A., \& Sofue, Y. 1996, ApJ, 464, 738

Kamaya, H., Mineshige, S., Shibata, K., \& Matsumoto, R. 1996, ApJL, 458, L25

Katgert, P. 1969, A\&A, 1, 54

Kennicutt, Jr., R. C., Edgar, B. K., \& Hodge, P. W. 1989, ApJ, 337,761

Kim, K.-T., \& Koo, B.-C. 2000, ApJ, 529, 229

Koo, B.-C., Heiles, C., \& Reach, W. T. 1991, in The Interstellar Disk-Halo Connection in Galaxies, ed. H. Bloemen, IAU Symp. 144 (Kluwer Academic Publishers), 165 
Koo, B.-C., Heiles, C., \& Reach, W. T. 1992, ApJ, 390, 108 Lee, S.-W. 1998, Ph.D. Thesis, Queen's University, Kingston, Ontario, Canada

Lonsdale Persson, C. J., \& Helou, G. 1987, ApJ, 314, 513

Mac Low, M.-M., \& McCray, R. 1988, ApJ, 324, 776

McCray, R., \& Kafatos, M. 1987, ApJ, 317, 190

Meyerdierks, H. 1991, A\&A, 251, 269

Mezger, P. G., \& Henderson, A. P. 1967, ApJ, 147, 471

Mirabel, I. F. 1982, ApJ, 256, 120

Norman, C. A., \& Ikeuchi, S. 1989, ApJ, 345, 372

O'Connell, R. W., Gallagher III, J. S., Hunter, D. A., \& Colley, W. N. 1995, ApJL, 446, L1

Paczyński, B. 1998, ApJ, 494, L45

Pildis, R. A., Bregman, J. N., \& Schombert, J. M. 1994, ApJ, 423, 190

Rand, R. J. 2000, ApJ, 537, L13

Rand, R. J., Kulkarni, S. R., \& Hester, J. J. 1990, ApJ, 352, L1
Rand, R. J., Kulkarni, S. R., \& Hester, J. J. 1992, ApJ, 396, 97

Rand, R. J., \& Stone, J. M. 1996, AJ, 111, 190

Rand, R. J., \& van der Hulst, J. M. 1993, AJ, 105, 2098

Santillán, A., Franco, J., Martos, M., \& Kim, J. 1999, ApJ, 515,657

Shapiro, P. R., \& Field, G. B. 1976, ApJ, 205, 762

Sofue, Y., Wakamatsu, K.-I., \& Malin, D. F. 1994, AJ, 108, 2102

Tomisaka, K., Habe, A., \& Ikeuchi, S. 1981, Ap\&SS, 78, 273

Wang, Q. D., Walterbos, R. A. M., Steakley, M. F., Norman, C. A., \& Braun, R. 1995, ApJ, 439, 176

Westerlund, B. E., \& Mathewson, D. S. 1966, MNRAS, 131, 371

Young, J. S., \& Scoville, N. Z. 1984, ApJ, 287, 153 